\title{
Exosomes Derived from Nanocomplex-Loaded Macrophages for Targeted Delivery of Docetaxel and siPLK1 against Castrate-Resistance Prostate Cancer
}

\section{Jing Tian}

Changhai Hospital

\section{Zongguang Tai}

Shanghai Skin Disease Hospital https://orcid.org/0000-0001-5603-745X

\section{Wei Zhang}

Tongji University Affiliated Shanghai Pulmonary Hospital

\section{Xiaoyu Wang}

Changhai Hospital

\section{Zhongjian Chen}

Shanghai Skin Disease Hospital

Qin Yu

Shanghai Skin Disease Hospital

\section{Guorui Li}

Changhai Hospital

\section{Chunai Gong}

Changhai Hospital

\section{Quangang Zhu}

Shanghai Skin Disease Hospital

\section{Yuan Gao}

Fudan University

Shen Gao ( $\square$ liullk@126.com )

Changhai Hospital https://orcid.org/0000-0002-5125-1606

\section{Research}

Keywords: Exosomes, Nanocomplex, Macrophages, Targeted Delivery, Docetaxel, Prostate Cancer Posted Date: August 17th, 2020 
License: (c) (i) This work is licensed under a Creative Commons Attribution 4.0 International License. Read Full License 


\section{Abstract}

Background. As a class of naturally occurring nanoparticles with low immunogenicity and high biocompatibility, exosomes have become a promising drug carrier for cancer therapy. However, their clinical applications remain a challenge due to their unsuitable donors, low scalability, as well as insufficient targeting ability. Here, we describe and validate a new strategy for drug loading into exosomes. We developed a folate-conjugated exosome (Co-Exo-FA) derived from nanocomplex-loaded Raw264.7 macrophages. This Co-Exo-FA containing docetaxel (DTX) and PLK1 siRNA (siPLK1) could be used for targeted therapy of castrate-resistance prostate cancer (CRPC).

Results. Our results showed that Co-Exo-FA exhibited high stability, enhanced cancer targeting ability, and led to the suppression of tumor growth with reduced toxicity in vivo. Moreover, the delivery of siPLK1 and DTX using an exosome system effectively silenced the PLK1 gene and exhibited improved anticancer effects.

Conclusion. Our results indicated that we managed to overcome major barriers to the efficient utility of exosomes and demonstrated the synergistic efficacy of siPLK1 and DTX in the treatment of CRPC, highlighting their potential value in therapeutic clinical applications.

\section{Background}

Prostate cancer is a commonly occurring tumor that poses severe risks to the health of men ${ }^{[1]}$. Endocrine therapy is the first-line treatment for advanced prostate cancer; however, most patients will progress to castration-resistant prostate cancer (CRPC). Accordingly, CRPC may exhibit strong resistance to chemotherapy, leading to disease progression with the associated deterioration and poor prognosis of patients ${ }^{[2]}$. Therefore, treatment of advanced CRPC is exceptionally challenging.

In the past 20 years, the development and implementation of nanodrug delivery systems have been hot topics in the field of cancer treatment. Regarding the therapeutic approaches for prostate cancer, studies have demonstrated that nanodrug delivery technology can make full use of the enhanced infiltration and retention (EPR) effects shown by tumors to achieve passive targeted delivery of drugs on site, so as to achieve greater efficacy and reduce the total effective dose and any side effects ${ }^{[3]}$. For example, invisible polymers, such as hydrophilic polyethylene glycol (PEG), are connected to the surface of hydrophobic nanoparticles to improve the stability of the nanosystem by changing the polarity of the nanoparticle surface ${ }^{[4]}$. At the same time, nanomaterials modified with PEG were shown able to avoid clearance by the reticular endothelial system (RES) and extend their retention time in the circulatory system. Thus, the combined application of the EPR effect and RES escape could improve the targeted delivery capacity of tumor drugs in nanodrug delivery systems ${ }^{[5]}$. Moreover, it could also provide increased predictability of the behavior of the nanodrug, which is particularly essential in the complex in vivo environment. 
Although a large number of preclinical studies have shown that nanodrug delivery systems have great potential in the field of tumor therapy, their clinical implementation still faces many difficulties. Many common factors limit the clinical applications of nanotherapeutic drugs, including the overcomplexity of the composition of the nanosystems, poor stability of drugs, poor biocompatibility, and nondegradability ${ }^{[6]}$, as well as the accumulation and potential toxicity of nanomaterials in essential organs. Another critical factor is that many nanoscale drug delivery systems rely highly on the EPR effect, which is considered passive targeting. However, the EPR effect provides some specificity, offering $20-30 \%$ increases in drug delivery ${ }^{[7]}$, with the effects varying across different tumors, thus making it difficult to predict the accumulation of drugs in targeted tumors.

Tumor cells often overexpress surface markers not present in healthy cells. As such, these tumor-specific antigen markers have been the basis of all approaches exploiting specific ligand targeting of tumor cells. The current consensus is that the recognition of tumor cell-specific surface receptors can be used in delivering therapeutic drugs to diseased cells ${ }^{[8,9]}$. The emergence of nanotechnology and its combination with oncological practices has excellent potential in the design and development of smarter and more efficient drug delivery systems ${ }^{[10]}$. The surface of a nanocarrier can be connected to a variety of targeted ligands, including antibodies ${ }^{[11]}$, aptamers ${ }^{[12]}$, or small-molecule ligands ${ }^{[13]}$, so as to construct multifunctional nanocomplexes that could be widely used in tumor imaging and therapy.

In order to address specific problems faced by the current nanodrug delivery technology, we selected the macrophage-derived exosome system, which has unique advantages as the drug carrier of choice. Exosomes are a kind of membranous vesicles with a diameter of about $100 \mathrm{~nm}$ released into the extracellular matrix after the multivesicular body (MVB) is fused with the cell membrane. The membranes of exosomes, like those of cells, have a stable phospholipid bilayer containing RNA, protein, DNA fragments, and other intracellular substances ${ }^{[14]}$. Exosomes are produced by various immune cells, epithelial cells, tumor cells, and most other types of cells, and secreted exosomes can enter the blood circulation, tissue fluids, and other bodily fluids, reaching other tissues and cells to exert their regulatory effects remotely [15]. The vesicle of an exosome can be loaded with a large amount of water-soluble substances, whereas the lipophilic region between the phospholipid bilayer can wrap hydrophobic substances [16]. Due to their unique structure and homology with somatic cells, exosomes can avoid clearance by the circulatory system and degradation in the extracellular environment [17]. Moreover, the presence of lipids and special proteins on their surface can allow for rapid fusion with target cells, delivering their loaded drugs after initial recognition and binding [16].

Exosomes can be loaded with drugs in two main ways: passive loading and active loading. Passive loading refers to the transfer of drugs into maternal cells or the cultivation of maternal cells with drugs, where the exosomes produced by these maternal cells will contain the drugs. Active loading refers to the loading of drugs into exosomes using physical methods, such as ultrasound, electroporation, extrusion, and freeze-thaw techniques $[18,19]$. The drug loading efficiency using these physical operations varies, 
but generally, it results in a certain degree of damage to the exosome, thus affecting the integrity of the exosome structure and function.

In this study, we used passive loading of exosomes by transfecting Raw264.7 mouse macrophage cells with docetaxel and siPLK1 co-loaded micelles. To the best of our knowledge, this was the first time that passive loading was used to directly produce exosomes containing chemotherapeutic drugs and siRNA. Folate-PEG was then conjugated to the produced exosomes to enhance their tumor accumulation (Fig. 1). We demonstrated that our exosomes exhibited proper prostate cancer targeting and anti-prostate cancer ability both in vitro and in vivo, as well as reducing the systemic toxicity of docetaxel (DTX).

\section{Results And Discussion}

\section{Extraction and drug determination of exosomes}

The SHRss micelles used for drug loading were synthesized using disulfide cross-linked stearyl-peptides containing arginine, histidine, and cysteine, according to our previous report ${ }^{[20]}$. Further, DTX-loaded, and DTX/siPLK1 co-loaded SHRss micelles (Co-PMs) were prepared using the probe-based ultrasonication technique.

Before the addition of Co-PMs to Raw264.7 cells, the sensitivity of Raw264.7 cells to DTX and Co-PMs was evaluated by performing a $24 \mathrm{~h}$ cytotoxicity assay (Fig. 2). As expected, Raw264.7 cells were shown to be extremely resistant to DTX and Co-PMs within the $24 \mathrm{~h}$ period. A low percentage of cell death was observed at the concentration of $8000 \mathrm{ng} / \mathrm{mL}$ DTX, whereas the $\mathrm{IC}_{50}$ value of DTX in various tumor cells

has been reported as $50-700 \mathrm{ng} / \mathrm{mL}^{[21-23]}$. Thus, it was suggested that DTX would not affect the viability of Raw264.7 cells at the administered dose of 2500 or $5000 \mathrm{ng} / \mathrm{mL}$.

As already known, cell contents usually appear in the released exosomes [14], and since Co-PMs contain both DTX and chemically modified stable siPLK1, it was expected that Raw264.7 cells that have accumulated Co-PMs, could release DTX and siPLK1 with their exosomes. Accordingly, we confirmed the presence of DTX and siPLK1 in exosomes produced by Raw264.7 cells.

Fig. 3A shows the DTX-loaded in exosomes as detected by HPLC analysis. DTX loading in exosomes obtained from cells treated with Co-PMs increased significantly compared with exosomes from cells treated with DTX alone, whether at concentrations of 2500 or $5000 \mathrm{ng} / \mathrm{mL}$. After linking DSPE-PEG-FA, the DTX content in the Co-Exo-FA slightly decreased, which might have been due to the release of DTX from the Co-Exo during the procedure. As confirmed in our previous studies ${ }^{21}$, SHRss micelles have been demonstrated to be able to deliver more chemotherapy drugs into cells. Therefore, more DTX was delivered into macrophages by Co-PMs to result in the secretion of exosomes containing more DTX. On the other hand, Co-PMs encapsulate DTX within the micelles, reducing the intracellular free DTX and thereby reducing the total DTX excreted by the powerful P-gp efflux pathway of the macrophage $[24,25]$. 
Moreover, the amount of siPLK1 in the exosomes secreted by Raw264.7 cells transfected with co-loaded SHRss micelles was determined by qRT-PCR analysis. As shown in Fig. 3B, the amount of siPLK1 increased with the increase in the transfection dose, as compared with controls. Since free siPLK1 cannot be easily absorbed by cells, exosomes produced by Raw264.7 cells treated with free siPLK1 contained only a small amount of SiPLK1. In order to ensure the stability of siPLK1 during endocytosis and exosome secretion, we chose chemically modified siPLK1, which is not easily degraded by enzymes and can remain stable in cells for an extended period of time [26]. This result demonstrated that exosomes secreted from Raw264.7 cells that had incorporated DTX/siPLK1 co-loaded SHRss micelles contained intact siPLK1.

\section{Ultrastructural analysis of exosomes}

Raw264.7 cells with internalized Co-PMs were incubated with serum-free medium for $24 \mathrm{~h}$ to trigger the release of exosomes. Exosomes were extracted from the conditioned medium using the differential centrifugation protocol. Dynamic light scattering (DLS) analysis indicated that the hydrodynamic diameter of Co-Exo-FA (153 nm) was a little longer than that of Co-Exo and blank Exo (Fig. 4A, 4B). The measured particle size was in good agreement with that obtained by transmission electron microscopy (TEM) imaging. Similarly, the zeta potential detected for Co-Exo-FA decreased from $-11.2 \mathrm{mV}$ to $-16.3 \mathrm{mV}$ compared to Co-Exo, and this change might be caused by the presence of the negatively charged PEG-FA.

Exosomes were also observed using TEM, and results indicated that exosomes regularly exhibited a spherical shape with a complete double-layer membrane structure (Fig. 4C). Likewise, the various Co-Exo exhibited no significant difference compared to blank Exo, whereas the edges of the Co-Exo-FA appeared to be more blurred. This indicated that PEG-FA was successfully distributed around the exosomes, and

thus could help exosomes escape clearance by the reticuloendothelial system (RES), as well as facilitate better tumor targeting.

In addition, the marker proteins of the exosomes were detected by western blotting analysis. As shown in Fig. 4D, CD9 molecule (CD9), CD63, CD81, and tumor susceptibility gene 101 (TSG101), which are exosome-specific proteins (exosome markers), were clearly detected in exosomes as compared to the respective levels in Raw264.3 cells. Moreover, calnexin-1, an endoplasmic reticulum marker protein was only detected in the Raw264.3 cell debris.

\section{Co-Exo-FA targeting of prostate cancer cells in vitro}

To study the cellular uptake of exosomes, DU145 and PC-3 cells were incubated with exosomes labeled with Nile red and FAM-siPLK1 for $4 \mathrm{~h}$ before analysis using flow cytometry. As shown in Fig. 5, there was an enhanced uptake of Co-Exo-FA by PC-3 and DU145 cells compared with unmodified Exo after $4 \mathrm{~h}$ of incubation, indicating that the PEG-FA modification contributed to the cellular uptake of Co-Exo-FA.

After $4 \mathrm{~h}$ of incubation, the qualitative internalization of Co-Exo-FA and their intracellular localization in PC-3 cells were evaluated using confocal laser scanning microscopy (CLSM) (Fig. 6). Blue fluorescence 
revealed nuclei stained by DAPI, and red fluorescence was due to the intracellular Nile red dye, whereas green fluorescence marked the internalized FAM-siPLK1.

In accordance with our flow cytometry analysis, both red (Nile red) and green (FAM-siPLK1) fluorescence was stronger in the Co-Exo-FA treated group relative to other groups, demonstrating the excellent targeting properties of Co-Exo-FA. In the merged view, the overlay of red and green fluorescence formed a yellow fluorescence, indicating that Nile red and FAM-siPLK1 were effectively co-delivered into PC-3 cells. On the other hand, the presence of widespread red and green fluorescence showed that part of the contents dissociated from the Co-Exo-FA upon delivery and spread into the cells.

Good biocompatibility of the membrane surface is crucial for the potential application of nanoparticles. As expected, the internalization mechanisms of free DTX and drug-loaded exosomes were different. Internalization of DTX was driven via passive diffusion, whereas the mechanisms of cellular uptake of exosomes were clathrin-mediated endocytosis and macropinocytosis [27]. Moreover, it is known that the surface of prostate cancer cells carries folate receptors, and folate modifications have been verified to lead to increased cellular uptake of nanoparticles [28]. Based on the results obtained from both the flow cytometry analysis and CLSM, it was suggested that folate receptor-mediated endocytosis played a vital role in the enhanced internalization of Co-Exo-FA. Above all, the most important outcome was that both DTX and siPLK1 were effectively co-delivered by Co-Exo-FA in vitro.

\section{Effects on cell viability and apoptosis}

To investigate the capacity of exosomes to influence the viability of prostate cancer cells, PC-3 and DU145 cells were treated with different exosome subtypes, and their viability was measured at $48 \mathrm{~h}$. As shown in Fig. 7, blank exosomes did not affect the viability of DU145 and PC-3 cell lines, suggesting high compatibility and low cytotoxicity. Nevertheless, the viability of PC-3 and DU145 cells was obviously inhibited by either drug-loaded exosomes or DTX alone, in a concentration-dependent manner. PC-3 and DU145 cells treated with DTX-Exo had a lower IC ${ }_{50}(38.5 \mathrm{ng} / \mathrm{mL}$ and $26.1 \mathrm{ng} / \mathrm{mL}$, respectively) than cells treated with DTX alone (39.8 ng/mL and $31.6 \mathrm{ng} / \mathrm{mL}$, respectively), which might be due to the different cellular uptake between chemotherapeutic drugs and drug-loaded nanoparticles and the sustained release of DTX from the exosomes. Interestingly, co-loading of siPLK1 achieved a synergistic effect, which enhanced the cytotoxicity of DTX-Exo $\left(\mathrm{IC}_{50}: 33.2 \mathrm{ng} / \mathrm{mL}\right.$ and $19.7 \mathrm{ng} / \mathrm{mL}$, respectively) in both cell lines. Among them, Co-Exo-FA exhibited the sharpest viability decrease $\left(\mathrm{IC}_{50}: 22.1 \mathrm{ng} / \mathrm{mL}\right.$ and 17.1 $\mathrm{ng} / \mathrm{mL}$, respectively), indicating that the introduction of folate contributed to the reduction of cell viability by approximately $33.4 \%$ in PC-3 and $13.2 \%$ in DU145 cells.

Annexin V-FITC/Propidium lodide (PI) staining and flow cytometry analysis were used to evaluate the effect of the various exosome subtypes on cell apoptosis (Fig. 8). Results showed the untreated cells and blank Exo-treated cells did not show a noticeable change in overall apoptosis and necrosis (no more than $10 \%$ in both groups) (Fig. 8). In contrast, extensive cell apoptosis was observed in drug-treated groups in PC-3 cells, and this was consistent with previous reports that DTX can induce cell apoptosis and death. 
Moreover, the combined loading of siPLK1 and DTX into exosomes increased cell apoptosis by $31.2 \%$ in PC-3 cells, compared with DTX-Exo alone. In addition, a 55.5\% increase in cell apoptosis was observed in the Co-Exo-FA treated group, indicating that incorporation of the folate group in Co-Exo resulted in increased induction of cell apoptosis.

\section{Downregulation of the target gene}

PC-3 cells were treated with blank Exo, DTX, DTX-Exo, siPLK1 loaded Co-Exo, and Co-Exo-FA. Consecutively, the expression of PLK1 was analyzed by qRT-PCR. Fig. 9A shows that the PLK1 mRNA levels in cells treated with siPLK1 loaded Co-Exo, and Co-Exo-FA were significantly reduced compared to those in cells treated with blank Exo, DTX, and DTX-Exo. In particular, the expression of PLK1 mRNA was reduced by $33.3 \%$ and $69.7 \%$, respectively. We believe that siPLK1 was loaded in Co-Exo in sufficient quantities to subsequently downregulate the expression of PLK1 mRNA and that the folate enhanced this gene regulation effect at the translational level.

For western blotting analysis, the GAPDH housekeeping gene was used as a control. We explored the potential regulation of the PLK1 gene by exosomes that harbor siRNA (siPLK1) against PLK1. The protein levels of PLK1 were also downregulated in the Co-Exo-FA treated PC-3 cells, compared to other groups (Fig. 9B). These results demonstrated that Co-Exo downregulated PLK1 at translational and posttranscriptional levels. This finding was consistent with the results showing that Co-exosomes could function as therapeutic vesicles.

\section{Co-Exo-FA targeting of tumors in a murine xenograft model}

The in vivo tumor targeting performance of Co-Exo-FA was investigated in a murine xenograft model established by subcutaneously inoculating PC-3 cells into the right flanks of BABL/C nude mice. Blank exosomes and Co-Exo-FA were labeled with DIR, which is usually used as an indicator for tracing nanoparticles in vivo [29]. Next, using in vivo imaging systems, we observed the fluorescence of tumorbearing mice at 1, 4, 8, and $24 \mathrm{~h}$ after intravenous administration of DIR, Co-Exo, and Co-Exo-FA. As shown in Fig. 10A, the strong fluorescence of DIR was visualized in all mice subjected to intravenous injection. More specifically, $1 \mathrm{~h}$ after the injection, a strong DIR fluorescence signal was observed at the tumor site in the Co-Exo-FA/DIR injected mice, and this signal was found to be strongest at $8 \mathrm{~h}$ postadministration. The signal at the tumor site was much stronger than that in the rest of the tissues. Once the mice were injected with Co-Exo-FA/DIR, a large amount of DIR appeared to be still accumulated in the tumor even $24 \mathrm{~h}$ post-administration. On the other hand, only faint DIR fluorescence was observed at the site of the tumor in the free DIR injected mice, and this fluorescence signal was mainly noted in the liver and kidneys. The intensity of the DIR signal in the tumors of the free DIR-treated mice did not change with time. Mice injected with Co-Exo/DIR exhibited a slight fluorescence accumulation in the tumor site after injection, which might have been a result of the EPR effect.

Tumors and organs were resected and scanned for visualization of their DIR fluorescence signal $24 \mathrm{~h}$ post-administration. As shown in Fig. 10B, Co-Exo-FA/DIR strongly accumulated in tumors, but exhibited 
very low accumulation in the other organs, including the heart, lung, and kidneys. However, strong fluorescence intensity was observed in the liver and kidneys of mice in the free DIR-treated group, which was much higher than that in the tumor site.

These data indicated that the Co-Exo-FA formulation significantly improved the efficiency of tumor accumulation. The PEG present on the surface of exosomes provided protection from elimination by the RES; therefore, Co-Exo-FA could make full use of the EPR effect. Meanwhile, the folate also contributed to the cellular uptake of Co-Exo-FA. Thus, we believe that, consistent with previous findings, the folate contributed to the efficiency of the tumor targeting [28].

\section{Tumor growth inhibition in vivo}

Next, we evaluated the anticancer effects of Co-Exo-FA in in vivo tumor growth experiments. PC-3 cells were subcutaneously engrafted on the back of male BALB/c nude mice. Subsequently, Co-Exo-FA was intravenously injected every $4 \mathrm{~d}$. Tumor sizes and body weights in each group were monitored periodically.

As shown in Fig. 11A, intravenous injection of blank exosomes had no significant inhibiting effect in tumor growth compared with the phosphate-buffered saline (PBS)-injected group. However, DTX, DTXExo, Co-Exo, and Co-Exo-FA treated mice exhibited different levels of tumor growth inhibition. The most significant antitumor activity was observed in the Co-Exo-FA treated group. The average tumor volume at day 27 was $72.3 \mathrm{~mm}^{3}$ in the Co-Exo-FA group, which was 17.4-, 15.7-, 7.1-, 4.1-, and 2.8-fold smaller than that in groups treated with PBS, blank Exo, DTX, DTX-Exo, and Co-Exo, respectively. The data obtained from our tumor xenograft mouse model confirmed that co-loading of DTX and siPLK1 into exosomes resulted in a synergistic effect against prostate cancer in vivo. The isolated tumors at day 27 were significantly smaller in the Co-Exo-FA group than in other groups, consistent with the observed growth curve of tumor volume (Fig. 11B).

The body weight measurements were used as an indicator of systemic toxicity by Co-Exo-FA. Fig. 11C shows that during the experiment, no significant body weight loss was observed in any of the treated mice compared to control groups, except for mice that were systemically administered free DTX.

Therefore, it was suggested that DTX-loaded in exosomes could remarkably reduce the systemic toxicity of free DTX in vivo.

\section{Histological analyses}

Tumor tissues were further evaluated through hematoxylin and eosin (H\&E) staining and IHC staining. As shown in Fig. 11A, both the blank Exo-treated and the control group exhibited a high density of cells displaying intact structures, and both the nuclei and the cellular outline remained clearly visible. In contrast, karyolysis, karyorrhexis, and cell disruption were observed in the tissues of tumors treated with DTX, DTX-Exo, Co-Exo, and Co-Exo-FA. In particular, cells treated with Co-Exo-FA lost their nuclei and cytoplasm, becoming an amorphous material, thus indicating the most evident and significant signs of 
necrosis and apoptosis. H\&E staining was also used to evaluate the influence of the administered exosomes to essential organs, including the heart, liver, spleen, lung, and kidneys. Fig. 12A shows that there was little necrosis of vital organ tissues in all the Exo-treated groups. However, we observed necrosis in the liver tissue of mice in the free DTX group. Since DTX was loaded in exosomes, the majority of which localized in tumor tissues, only low-level systemic toxicity was expected.

To detect the expression of PLK1 protein in tumor tissues, we performed IHC staining. As shown in Fig. 12B, the expression of PLK1 was significantly reduced in the tumors treated with Co-Exo-FA compared with other groups. Meanwhile, the administration of free DTX or DTX-Exo showed no visible effect on the expression of PLK1.

By promoting tumor apoptosis and downregulating the expression of the PLK1 gene, this study revealed the synergistic antitumor effects of the Co-Exo-FA delivery system. These results confirmed that Co-ExoFA could function as an efficient chemotherapeutic and siRNA delivery system.

\section{Conclusions}

Herein, we report a series of experiments performed to determine the production, targeting, and therapeutic effects of a conjugate exosome (Co-Exo-FA) loaded with a chemotherapeutic drug and a PLK1 siRNA. We isolated Co-Exo with high efficiency using nanocomplexes loaded in Raw 264.7 macrophages. The FA targeting mode provided the Exo with precise targeting properties, confirmed both in vitro and in vivo. By delivering chemotherapeutic drugs and PLK1 siRNA, these functional exosomes were shown to enable effective tumor therapy in vivo. Our findings showed that Co-Exo-FA might facilitate the development of natural theragnostic nanoplatforms with the potential for combined therapies against cancer.

\section{Methods}

\section{Materials}

Docetaxel was obtained from Sangon Biotech (Shanghai, China). The chemically modified siPLK1 (sense: 5'-UGAAGAAGAUCACCCUCCUUATT-3', antisense: 5'-UAAGGAGGGUGAUCUUCUUCATT-3') was synthesized by GenePharma Co. Ltd. (Shanghai, China). Fetal bovine serum (FBS) and buffers, Dulbecco's modified eagle medium (DMEM), 0.25\% trypsin/EDTA, penicillin-streptomycin solution (5 $\mathrm{kU} / \mathrm{mL}$ ), and Trizol were purchased from Life Technologies (Grand Island, NY, USA). The BCA protein assay kit was obtained from Beyotime (Nanjing, China). The 4',6-diamidino-2-phenylindole (DAPI) was obtained from Sigma-Aldrich (St. Louis, MO, USA), and the 1,1'-dioctadecyltetramethyl indotricarbocyanine iodide (DIR) was purchased from Biotium (Hayward, CA, USA). The DSPE-PEG-FA was purchased from Ponsure Biological (Shanghai, China), and the Annexin V-FITC/PI Apoptosis Analysis Kit was purchased from BD Biosciences (San Diego, CA, USA). Antibodies were obtained from 
Abcam (Cambridge, MA, USA). All other reagents were of analytical grade and purchased from Weibian Co., Ltd. (Shanghai, China).

\section{Cell culture}

The DU145 and PC-3 human prostate cell lines and the RAW264.7 mouse macrophage-like cells were obtained from the China Centre for Type Culture Collection (CCTCC, Shanghai, China). Both DU145 and PC-3 cells were cultured in DMEM with 10\% FBS. Likewise, RAW264.7 cells were maintained in DMEM supplemented with $10 \%$ FBS and $1 \%$ penicillin/streptomycin. All cells were grown in an atmosphere containing $5 \% \mathrm{CO}_{2}$ and maintained at $37^{\circ} \mathrm{C}$ in a humidified chamber (Thermo Fisher Scientific, MA, USA).

\section{Animals}

Male BALB/c nude mice (weighing 14-15 g, housed in the Second Military Medical University Animal Care Center, Shanghai, China) were maintained under specific pathogen-free (SPF) conditions with free access to food and water. This study was performed in accordance with the National Institutes of Health Guide for the Care and Use of Laboratory Animals (NIH Publications No. 8023, revised 1978) and was approved by the Research Center for Laboratory Animals of the Second Military Medical University of China.

\section{Preparation of polymer micelles}

The self-assembling, disulfide cross-linked stearyl-peptide-based micellar system (SHRss) used for loading DTX and siPLK1 was synthesized as previously reported ${ }^{[20]}$. The DTX-loaded SHRss polymer micelles (DTX-PMs) were prepared using the probe-based ultrasonication technique ${ }^{[30]}$. Briefly, $5 \mathrm{mg}$ SHRss was dissolved in $5 \mathrm{~mL}$ of distilled water. The DTX was dissolved in $1 \mathrm{~mL}$ of dichloromethane, and the solution was dropped into the SHRss solution, followed by ultrasonication for $1 \mathrm{~min}$ at $200 \mathrm{~W}$ using a probe-based sonicator (JY92-IIN; Xinzhi Scientific Co., Ltd., Ningbo, China). After that, the mixed solution was stirred overnight at $25^{\circ} \mathrm{C}$ to evaporate the dichloromethane. Finally, the solution was filtrated through a membrane (pore size: $0.45 \mu \mathrm{m}$ ) to remove any insoluble DTX. To prepare DTX- and siPLK1-loaded SHRss polymer micelles (Co-PMs), siPLK 1 was added into DTX-PMs solution at N/P $=10$ followed by 30 $\mathrm{s}$ vortexing and $30 \mathrm{~min}$ incubation at $25^{\circ} \mathrm{C}$ before use.

\section{Preparation and purification of exosomes}

Exosomes were extracted using the sequential centrifugation method ${ }^{[31,32]}$. Briefly, Raw264.7 cells were seeded in culture flasks to reach $80-90 \%$ confluence. After replacing the culture medium, cells were washed with PBS solution. Then, Co-PMs containing DTX and siPLK1 were added into the culture flasks for uptake by macrophages. After $4 \mathrm{~h}$ incubation, the medium was replaced by DMEM containing $10 \%$ exosome-free FBS, and cells were cultured for an additional $24 \mathrm{~h}$. Subsequently, the culture medium was collected, and exosomes were concentrated by ultracentrifugation with a TY70Ti rotor (Beckman Coulter, Chaska, MN, USA) at $2000 \times \mathrm{g}$ for $20 \mathrm{~min}$ at $4{ }^{\circ} \mathrm{C}$, washed with cold PBS, and collected at $10000 \times \mathrm{g}$ for 
$30 \mathrm{~min}$ at $4{ }^{\circ} \mathrm{C}$ to remove cell debris. Supernatants were passed through $0.22 \mu \mathrm{m}$ membrane filters before being transferred into a special centrifuge tube for overspeed centrifugation at $120000 \times \mathrm{g}$ for $70 \mathrm{~min}$. Exosomes were collected and resuspended in PBS. The total protein content of exosomes was quantified by BCA assays as follows. Cells and exosomes were suspended in RIPA lysis buffer (Thermo Fisher Scientific) on ice for $30 \mathrm{~min}$. Then, lysates were centrifuged at $13000 \times \mathrm{g}$ for $20 \mathrm{~min}$, and protein concentration was analyzed using the BCA protein assay kit according to manufacturer's protocols. Equal amounts of protein from exosomes and cells were denatured by boiling for $5 \mathrm{~min}$, separated by SDSPAGE electrophoresis, and transferred onto a PVDF membrane (Millipore, Bedford, MA, USA). Membranes were blocked with $5 \%$ non-fat milk powder in Tris-buffered saline Tween 20 (TBS-T) for $1 \mathrm{~h}$ at $25^{\circ} \mathrm{C}$ with the following primary antibodies: rabbit monoclonal anti-CD9 (1:200), anti-CD63 (1:200), anti-CD81 (1:200), anti-TSG101 (1:250), and anti-calnexin-1 (1:200). After washing, membranes were incubated with goat anti-rabbit IgG-HRP secondary antibody (1:2000) for $90 \mathrm{~min}$. Following another wash, protein bands were visualized using an enhanced chemiluminescence ( $E C L)$ system (Millipore, Darmstadt, Germany).

\section{Characterization of exosomes}

The size and zeta potential of exosomes were characterized using the Zetasizer Nano ZS (Malvern; Westborough, MA, USA) system. The morphology of exosomes was examined by TEM. For TEM analysis of exosomes, one drop of a solution containing $10 \mu \mathrm{g} / \mathrm{mL}$ protein of exosomes was absorbed onto a copper grid coated with carbon film and stained with $2 \%$ uranyl acetate. Images were recorded under 75 kV acceleration voltage using a transmission electron microscope (Hitachi H-600, Shanghai, China).

\section{Loading of DTX and siPLK in exosomes}

The presence of DTX in exosomes was determined using a high-performance liquid chromatography system (Agilent LC-1260; Agilent, Santa Clara, CA, USA). Briefly, exosomes containing $100 \mu$ p protein were extracted by a Bond Elut LRC C-18 solid-phase extraction cartridge (Varian, Harbor City, CA, USA) followed by evaporation to dryness. Subsequently, exosomes were mixed with $0.6 \mathrm{~N}$ perchloric acid containing 5 $\mathrm{mg} / \mathrm{mL}$ sodium disulfite $(1: 1, \mathrm{v} / \mathrm{v})$, centrifuged, filtered through $0.22 \mu \mathrm{m}$ filters, and finally, $20 \mu \mathrm{L}$ was injected into the HPLC system. Chromatography was performed on a C18 column ( $4.6 \times 250 \mathrm{~mm}, 5 \mu \mathrm{m})$ at $40^{\circ} \mathrm{C}$ using acetonitrile and water $(60: 40, \mathrm{v} / \mathrm{v})$ as mobile phase pumped at a rate of $1.0 \mathrm{~mL} / \mathrm{min}$. The detecting condition in the UV-visible detector was set at $230 \mathrm{~nm}$. The concentration of DTX in exosomes was expressed as ng of DTX per mg of protein.

To quantify siPLK1, total RNA was extracted from the Exo-rich fraction using Trizol, following treatment with DNase I, and according to the protocol of the supplier. The RNA concentration was determined by UV spectrophotometry. Reverse transcription reactions of $10 \mu \mathrm{g}$ RNA were performed using the Transcriptor First Strand cDNA Synthesis Kit (Roche, Switzerland). The PCR primer used for siPLK1 was synthesized by GenePharma Co. Ltd. (Shanghai, China). qRT-PCR analysis was performed using the FastStart Universal SYBR Green Master (ROX) (Roche, Switzerland). The qRT-PCR reaction is defined at 40 cycles as the fractional cycle number at which the fluorescence passes a fixed threshold. The concentration of RNA was then calculated using RNA of known concentration added as an internal control. 


\section{Cellular uptake assay}

For the cellular uptake study, PC-3 cells were seeded in 6-well plates at a density of $1.5 \times 10^{5}$ cells per well and cultured for $24 \mathrm{~h}$. The DTX in exosomes was replaced by Nile red dye, and the siPLK1 loaded in exosomes was labeled with FAM dye. Exosomes were extracted and added to the cultured PC-3 cells. Final concentrations of the FAM-siPLK1 and Nile red-loaded exosomes were $100 \mathrm{nM}$ and $50 \mathrm{ng} / \mathrm{mL}$, respectively. After $4 \mathrm{~h}$ incubation, the medium was removed, and cells were washed and trypsinized. Then, the cellular uptake of Nile red and FAM-siPLK1 was detected using a flow cytometer (FACSCalibur; BD Biosciences, UK). In addition, a cellular uptake assay was also performed using CLSM as follows. PC3 cells were seeded in a glass-covered 24-well plate at a density of $2.5 \times 10^{4}$ cells per well and cultured for $24 \mathrm{~h}$. Then, exosomes loaded with FAM-siPLK1 and Nile red were added to the PC-3 cells. After $4 \mathrm{~h}$ of incubation, cells were washed three times with PBS and fixed with $4 \%$ paraformaldehyde for 30 min, followed by incubation with DAPI (4',6-diamidino-2-phenylindole) working solution for 20 min at $25^{\circ} \mathrm{C}$. After washing with PBS, cells were sealed and visualized by CLSM (Leica, Germany).

\section{Cell viability assay}

The cytotoxicity of our conjugated exosome (Co-Exo) was assessed in PC-3 and DU145 cells. Briefly, PC-3 and DU145 cells were seeded in 96-well plates (5000 and 4000 cells per well, respectively) and allowed to grow for $24 \mathrm{~h}$. The medium was then replaced with culture medium containing either DTX, DTX-Exo, CoExo, Co-Exo-FA, or empty exosomes (Exo) at various concentrations. After incubation for $48 \mathrm{~h}, 10 \mu \mathrm{L}$ of CCK-8 solution was added to each well and incubated for $1.5 \mathrm{~h}$. Absorbance at $450 \mathrm{~nm}$ was read on a microplate reader (Thermo Fisher Scientific). Cell viability (\%) was calculated, and data were expressed as mean \pm SD of 6 replicate wells.

\section{Flow cytometry apoptosis assay}

PC-3 cells were seeded in 12-well plates $\left(1 \times 10^{5}\right.$ cells per well $)$ and incubated for $24 \mathrm{~h}$ in $5 \% \mathrm{CO}_{2}$ at $37^{\circ} \mathrm{C}$. After treatment with either DTX, DTX-Exo, Co-Exo, or Co-Exo-FA for $24 \mathrm{~h}$, cells were trypsinized, collected, and washed. Cells were stained with Annexin V-FITC and PI stock solutions according to the manufacturer's protocol. Samples were analyzed using an analytical flow cytometer (FACSCalibur). Untreated cells were used as background control.

\section{Proteins and mRNA assay}

PC-3 cells were seeded in 6-well plates $\left(2 \times 10^{5}\right.$ cells per well) and cultured for $24 \mathrm{~h}$. Cells were treated with either DTX, DTX-Exo, Co-Exo, or Co-Exo-FA. After additional $48 \mathrm{~h}$ incubation, cells were harvested and suspended in RIPA lysis buffer on ice for $30 \mathrm{~min}$. Then, the PLK1 mRNA was analyzed by qRT-PCR, and PLK1 proteins were analyzed by western blotting assay, as previously described. The primer sequences were used as follows: PLK1 5'-TGACTCAACACGCCTCATCC-3' (forward) and 5'GCTCGCTCATGTAATTGCGG-3' (reverse); $\beta$-actin 5'-GTGGACATCCGTAAGGACCTGTACG-3' (forward) and 5'-CTCCTGCTTGCTGATCCACATCTGC-3' (reverse). 


\section{In vivo targeting and biodistribution}

To establish a mouse tumor xenograft model, $0.2 \mathrm{~mL}$ of PC-3 cell suspension $\left(1 \times 10^{7}\right.$ cells $)$ was inoculated subcutaneously into the right flank of male BALB/C nude mice (4 wk). For the in vivo distribution study, a near-infrared fluorescein DIR was used as a model drug in the performed assays. When tumors had grown to $100 \mathrm{~mm}^{3}$ in size, mice were injected with either free DIR, DIR-Exo or DIR-ExoFA via the tail vein at a single DIR dose of $50 \mu \mathrm{g} / \mathrm{kg}$, and then imaged at 1, 4, 8, and 24 h post injection at $748 \mathrm{~nm}$ excitation and $780 \mathrm{~nm}$ emission using the Xenogen IVIS-200 imaging system (Caliper Life Sciences, Hopkinton, MA, USA). Mice were sacrificed $24 \mathrm{~h}$ after administration, and tumors, as well as other vital organs, were resected and immediately imaged.

\section{In vivo antitumor effects}

Three days after injection of tumor cells, the mice were randomly divided into five groups (six mice in each group) and intravenously injected with either PBS, DTX, DTX-Exo, Co-Exo, or Co-Exo-FA at a DTX equivalent dose of $6 \mathrm{mg} / \mathrm{kg}$. The injection was performed every $4 \mathrm{~d}$ up to 5 times. The body weight of each mouse and the longest and shortest diameter of xenograft tumors were measured every $3 \mathrm{~d}$. Mice were euthanized on day 27 . The weight of each tumor was determined with a microbalance, and tumors were subsequently fixed for histological analysis.

\section{Histological analyses}

Tumors and organs were fixed in $4 \%$ paraformaldehyde for $24 \mathrm{~h}$ before paraffin embedding. Tissue slices from tumors and organs were stained with H\&E for observation. Immunostaining was performed according to standard protocols. Rabbit monoclonal antibodies against PLK1 were used as primary antibodies, and a biotinylated goat anti-rabbit immunoglobulin was used as the secondary antibody. For immunohistochemistry ( $\mathrm{IHC})$, sections were incubated with the primary antibody at $25^{\circ} \mathrm{C}$ for $2 \mathrm{~h}$ and then incubated with the biotinylated goat anti-rabbit antibody for $20 \mathrm{~min}$. Sections were processed using a VECTASTAIN ABC Kit and finally stained with hematoxylin. Images were taken using a microscope (Nikon E-800M; Nikon Corporation, Japan).

\section{Statistical analysis}

All values are shown as mean \pm SD. One-way ANOVA or Student's t-test were used to establish statistical significance. A P-value $<0.05$ was considered statistically significant.

\section{List Of Abbreviations}

folate-conjugated exosome, Co-Exo-FA; docetaxel, DTX; PLK1 siRNA, siPLK1; castrate-resistance prostate cancer, CRPC; polyethylene glycol, PEG; reticular endothelial system, RES; enhanced infiltration and retention, EPR. 


\section{Declarations}

None.

\section{Ethics approval and consent to participate}

The experimental protocol was established, according to the ethical guidelines of the Changhai Hospital.

\section{Consent for publication}

Not applicable.

\section{Availability of data and materials}

The datasets used and/or analyzed during the current study are available from the corresponding author on reasonable request.

\section{Competing interests}

The authors declare that they have no competing interests

\section{Funding}

This work was supported by the National Natural Science Foundation of China (NSFC 81803078, 81703051, 81472394, 81703434 and 81703059).

\section{Authors' contributions}

The manuscript was written through contributions of all authors. All authors have given approval to the final version of the manuscript. Jing Tian, Zongguang Tai, Wei Zhang, contributed equally.

\section{Acknowledgments}

Not applicable.

Corresponding author

Tel./fax: +86 21 81873715, liullk@126.com (S.Gao)

Tel./fax: +86 21 58980025, yuan_gao@fudan.edu.cn (Y. Gao)

Tel./fax: +86 21 61833160, qgzhu@126.com (Q. Zhu)

\section{References}

1. Siegel RL, Miller KD, Jemal A: Cancer statistics, 2019. CA Cancer J Clin 2019, 69:7-34. 
2. Clegg NJ, John W, Joseph JD, Chris T, Samedy O, Anna D, Yu C, Kate G, Bischoff ED, Ling C: ARN-509: a novel antiandrogen for prostate cancer treatment. Cancer Research 2012, 72:1494.

3. Maeda $\mathrm{H}$ : Toward a full understanding of the EPR effect in primary and metastatic tumors as well as

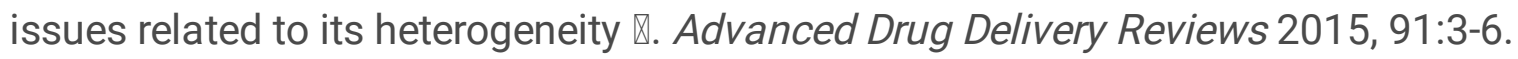

4. Liu Y, Shipton MK, Ryan J, Kaufman ED, Franzen S, Feldheim DL: Synthesis, stability, and cellular internalization of gold nanoparticles containing mixed peptide-poly(ethylene glycol) monolayers. Anal Chem 2007, 79:2221-2229.

5. Gabizon A, Martin F: Polyethylene glycol-coated (pegylated) liposomal doxorubicin. Rationale for use in solid tumours. Drugs 1997, 54 Suppl 4:15-21.

6. Hua S, de Matos MBC, Metselaar JM, Storm G: Current Trends and Challenges in the Clinical Translation of Nanoparticulate Nanomedicines: Pathways for Translational Development and Commercialization. Front Pharmacol 2018, 9:790.

7. Kobayashi H, Watanabe R, Choyke PL: Improving conventional enhanced permeability and retention (EPR) effects; what is the appropriate target? Theranostics 2013, 4:81-89.

8. Jahanban-Esfahlan R, Seidi K, Monhemi H, Adli ADF, Minofar B, Zare P, Farajzadeh D, Farajnia S, Behzadi R, Abbasi MM, et al: RGD delivery of truncated coagulase to tumor vasculature affords local thrombotic activity to induce infarction of tumors in mice. Sci Rep 2017, 7:8126.

9. Ruoslahti E, Bhatia SN, Sailor MJ: Targeting of drugs and nanoparticles to tumors. J Cell Biol 2010, 188:759-768.

10. Gmeiner WH, Ghosh S: Nanotechnology for cancer treatment. Nanotechnol Rev 2015, 3:111-122.

11. Cardoso MM, Peca IN, Roque AC: Antibody-conjugated nanoparticles for therapeutic applications. Curr Med Chem 2012, 19:3103-3127.

12. Yang L, Sun H, Liu Y, Hou W, Yang Y, Cai R, Cui C, Zhang P, Pan X, Li X, et al: Self-Assembled AptamerGrafted Hyperbranched Polymer Nanocarrier for Targeted and Photoresponsive Drug Delivery. Angew Chem Int Ed Engl 2018, 57:17048-17052.

13. El-Gogary RI, Rubio N, Wang JT, Al-Jamal WT, Bourgognon M, Kafa H, Naeem M, Klippstein R, Abbate $\mathrm{V}$, Leroux $\mathrm{F}$, et al: Polyethylene glycol conjugated polymeric nanocapsules for targeted delivery of quercetin to folate-expressing cancer cells in vitro and in vivo. ACS Nano 2014, 8:1384-1401.

14. Kilchert C, Wittmann S, Vasiljeva L: The regulation and functions of the nuclear RNA exosome complex. Nat Rev Mol Cell Biol 2016, 17:227-239.

15. Tkach M, Théry C: Communication by Extracellular Vesicles: Where We Are and Where We Need to Go. Cell 2016, 164:1226-1232.

16. Batrakova EV, Kim MS: Using exosomes, naturally-equipped nanocarriers, for drug delivery. J Control Release 2015, 219:396-405.

17. Wiklander OP, Nordin JZ, O'Loughlin A, Gustafsson Y, Corso G, Mager I, Vader P, Lee Y, Sork H, Seow Y, et al: Extracellular vesicle in vivo biodistribution is determined by cell source, route of administration and targeting. J Extracell Vesicles 2015, 4:26316. 
18. Tarasov VV, Svistunov AA, Chubarev VN, Dostdar SA, Sokolov AV, Brzecka A, Sukocheva O, Neganova ME, Klochkov SG, Somasundaram SG, et al: Extracellular vesicles in cancer nanomedicine. Semin Cancer Biol 2019.

19. JPK A, MM S: Strategic design of extracellular vesicle drug delivery systems. Advanced drug delivery reviews 2018, 130:12-16.

20. Tai Z, Wang X, Tian J, Gao Y, Zhang L, Yao C, Wu X, Zhang W, Zhu Q, Gao S: Biodegradable stearylated peptide with internal disulfide bonds for efficient delivery of siRNA in vitro and in vivo. Biomacromolecules 2015, 16:1119-1130.

21. Ernsting MJ, Tang WL, MacCallum N, Li SD: Synthetic modification of carboxymethylcellulose and use thereof to prepare a nanoparticle forming conjugate of docetaxel for enhanced cytotoxicity against cancer cells. Bioconjug Chem 2011, 22:2474-2486.

22. Yang X, Liu Y, Li W, Li A, Sun Q: DKK4-knockdown enhances chemosensitivity of A549/DTX cells to docetaxel. Acta Biochim Biophys Sin (Shanghai) 2017, 49:899-906.

23. Liu F, Feng L, Zhang L, Zhang X, Zhang N: Synthesis, characterization and antitumor evaluation of CMCS-DTX conjugates as novel delivery platform for docetaxel. Int J Pharm 2013, 451:41-49.

24. Shin D, Christie C, Ju D, Nair RK, Molina S, Berg K, Krasieva TB, Madsen SJ, Hirschberg H: Photochemical internalization enhanced macrophage delivered chemotherapy. Photodiagnosis Photodyn Ther 2018, 21:156-162.

25. Fu J, Wang D, Mei D, Zhang H, Wang Z, He B, Dai W, Zhang H, Wang X, Zhang Q: Macrophage mediated biomimetic delivery system for the treatment of lung metastasis of breast cancer. $J$ Control Release 2015, 204:11-19.

26. Ku SH, Jo SD, Lee YK, Kim K, Kim SH: Chemical and structural modifications of RNAi therapeutics. Adv Drug Deliv Rev 2016, 104:16-28.

27. Tian T, Zhu YL, Zhou YY, Liang GF, Wang YY, Hu FH, Xiao ZD: Exosome uptake through clathrinmediated endocytosis and macropinocytosis and mediating miR-21 delivery. J Biol Chem 2014, 289:22258-22267.

28. Kang MH, Yoo HJ, Kwon YH, Yoon HY, Lee SG, Kim SR, Yeom DW, Kang MJ, Choi YW: Design of Multifunctional Liposomal Nanocarriers for Folate Receptor-Specific Intracellular Drug Delivery. Mol Pharm 2015, 12:4200-4213.

29. Cash KJ, Li C, Xia J, Wang LV, Clark HA: Optical drug monitoring: photoacoustic imaging of nanosensors to monitor therapeutic lithium in vivo. ACS Nano 2015, 9:1692-1698.

30. Yao C, Liu J, Wu X, Tai Z, Gao Y, Zhu Q, Li J, Zhang L, Hu C, Gu F, et al: Reducible self-assembling cationic polypeptide-based micelles mediate co-delivery of doxorubicin and microRNA-34a for androgen-independent prostate cancer therapy. J Control Release 2016, 232:203-214.

31. Melo SA, Sugimoto H, O'Connell JT, Kato N, Villanueva A, Vidal A, Qiu L, Vitkin E, Perelman LT, Melo $\mathrm{CA}$, et al: Cancer exosomes perform cell-independent microRNA biogenesis and promote tumorigenesis. Cancer Cell 2014, 26:707-721. 
32. Alvarez-Erviti L, Seow Y, Yin H, Betts C, Lakhal S, Wood MJ: Delivery of siRNA to the mouse brain by systemic injection of targeted exosomes. Nat Biotechnol 2011, 29:341-345.

\section{Figures}

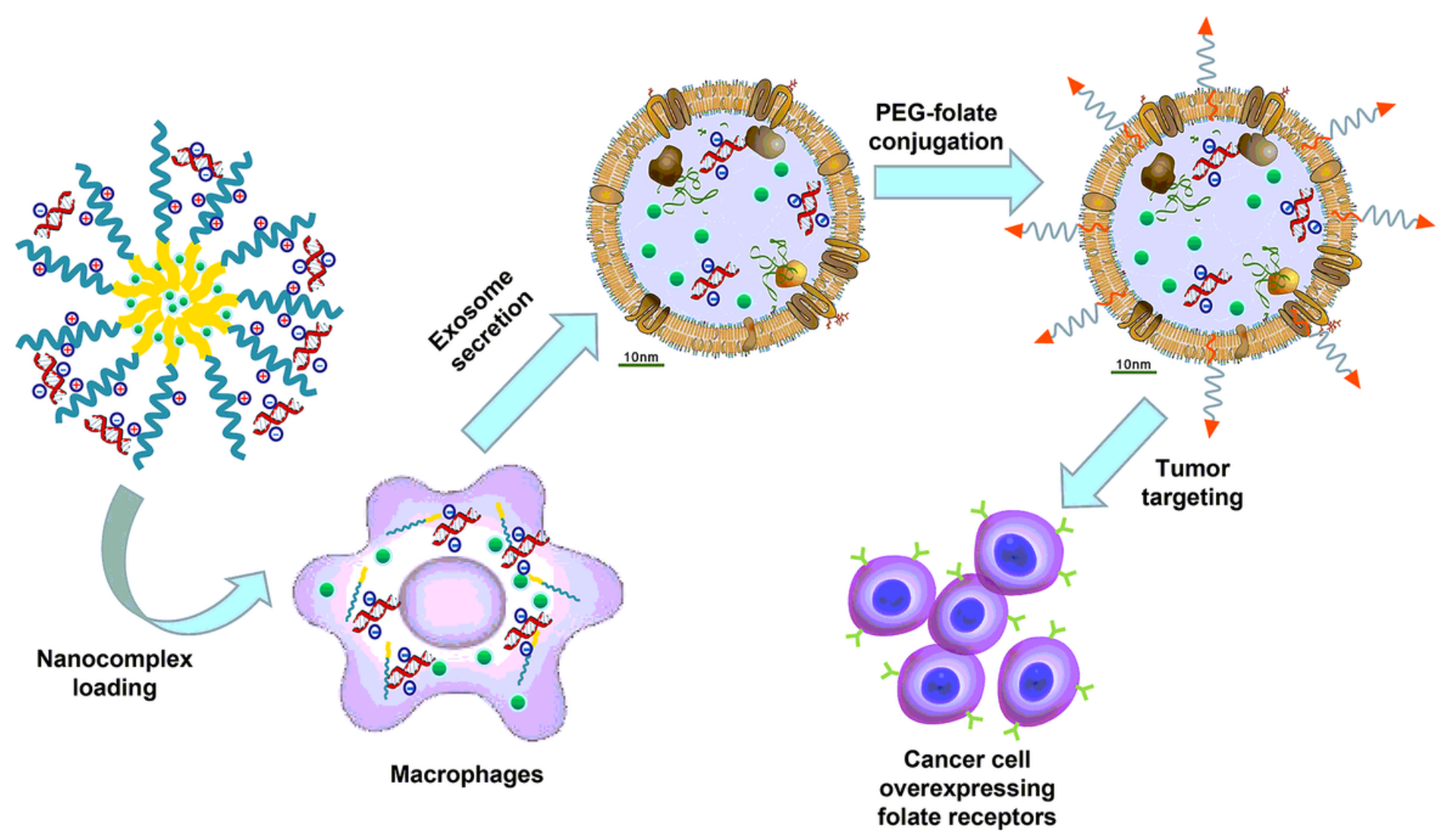

\section{Figure 1}

Scheme of the formation process of DTX and siPLK1-loaded exosomes. 


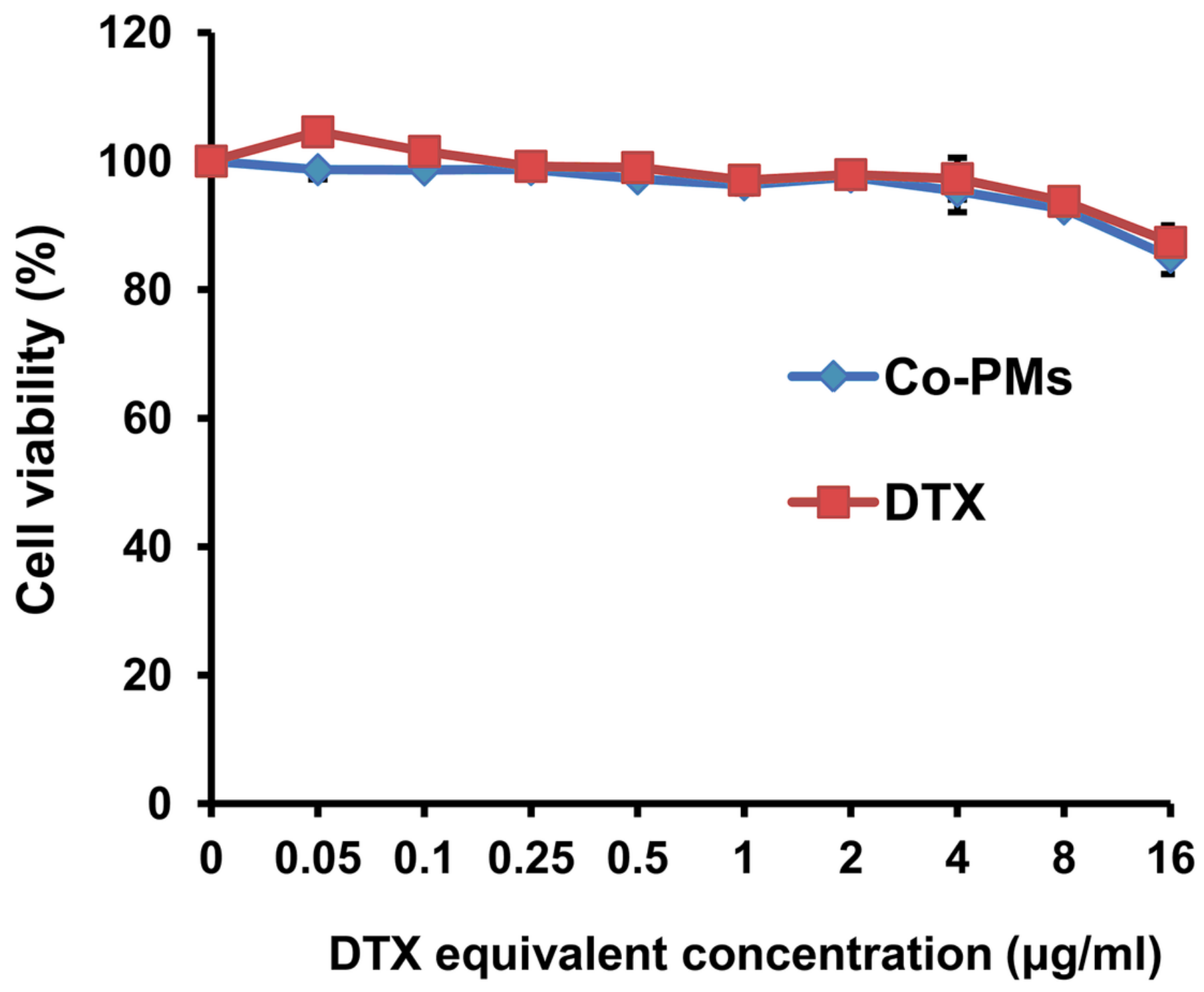

Figure 2

The sensitivity of Raw264.7 cells to DTX and Co-PMs. The sensitivity was determined as cell viability following $24 \mathrm{~h}$ treatment with DTX up to the dose of $16 \mu \mathrm{g} / \mathrm{mL}$. Data are shown as mean \pm SD of six independent tests. 
A

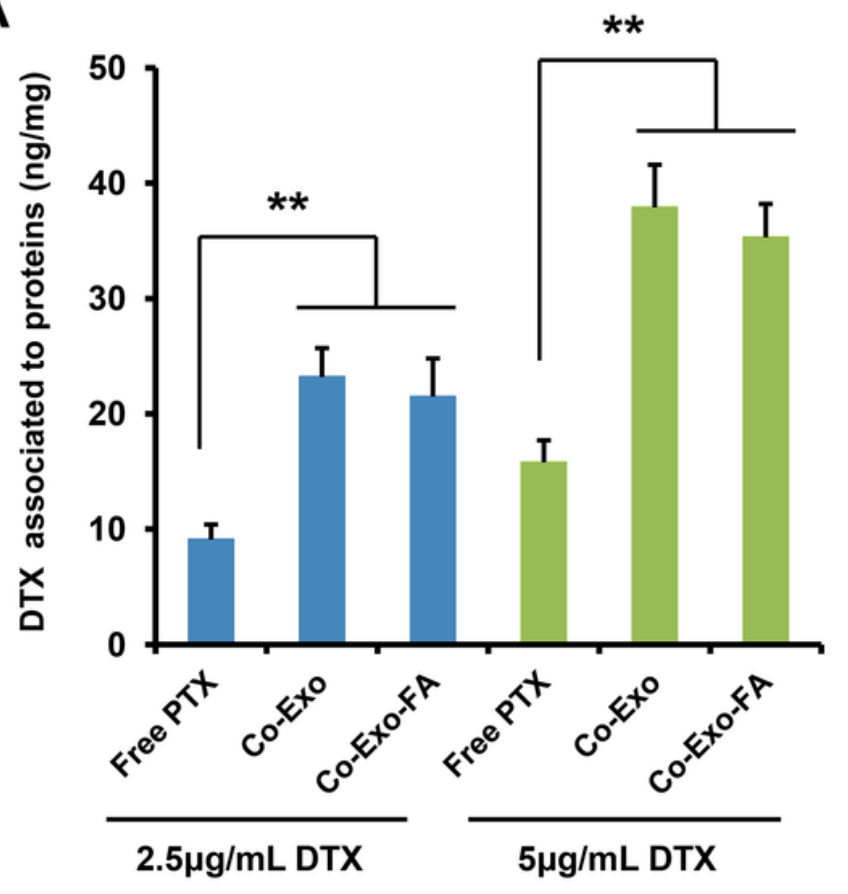

B

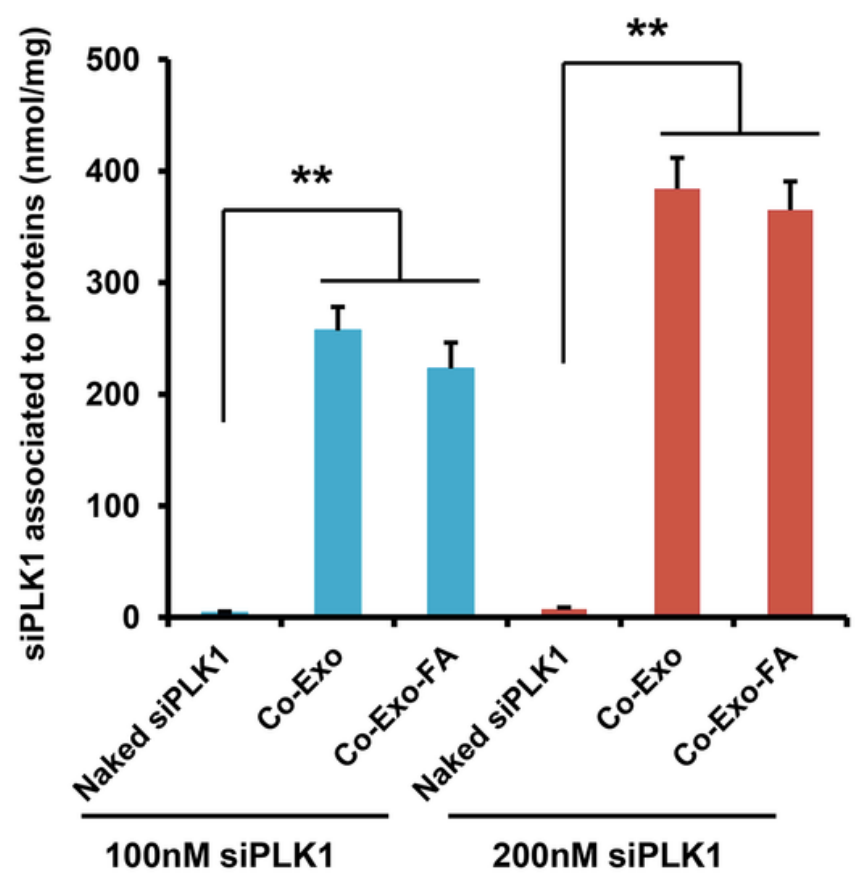

Figure 3

Comparison of DTX and siPLK1 levels in the exosomes secreted by Raw264.7 cells. (A) Release of DTX from exosomes secreted by Raw264.7 cells. Free DTX: cells were treated with DTX for $4 \mathrm{~h}$ and exosomes were collected after $24 \mathrm{~h}$; Co-Exo: cells were treated with DTX- and siPLK1-loaded SHRss polymer micelles (Co-PMs) for $4 \mathrm{~h}$ and exosomes were collected after $24 \mathrm{~h}$; Co-Exo-FA: Co-Exo were incubated with DSPEPEG-FA for $4 \mathrm{~h}$ and then purified. (B) Levels of siPLK1 in the exosomes secreted by Raw264.7 cells. Naked siPLK1: cells were treated with siPLK1 for $4 \mathrm{~h}$ and exosomes were collected after $24 \mathrm{~h}$; Co-Exo: cells were treated with DTX-and siPLK1-loaded SHRss polymer micelles (Co-PMs) for $4 \mathrm{~h}$ and exosomes were collected after $24 \mathrm{~h}$; Co-Exo-FA: Co-Exo were incubated with DSPE-PEG-FA for $4 \mathrm{~h}$ and then purified. Chemically modified siPLK1 was used in this experiment. Data are shown as mean \pm SD of three independent tests. ${ }^{\star *} \mathrm{P}<0.01$. 

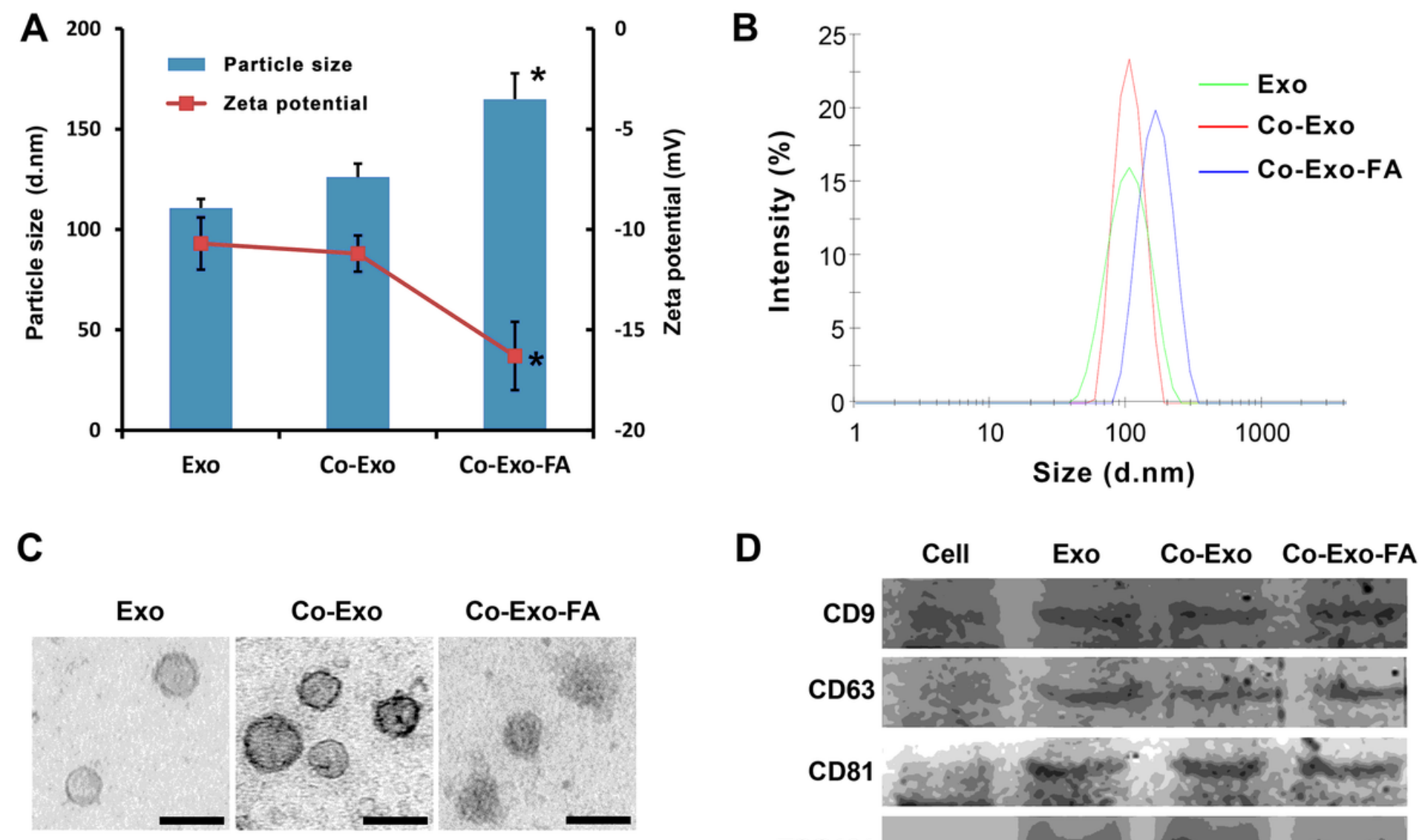

D

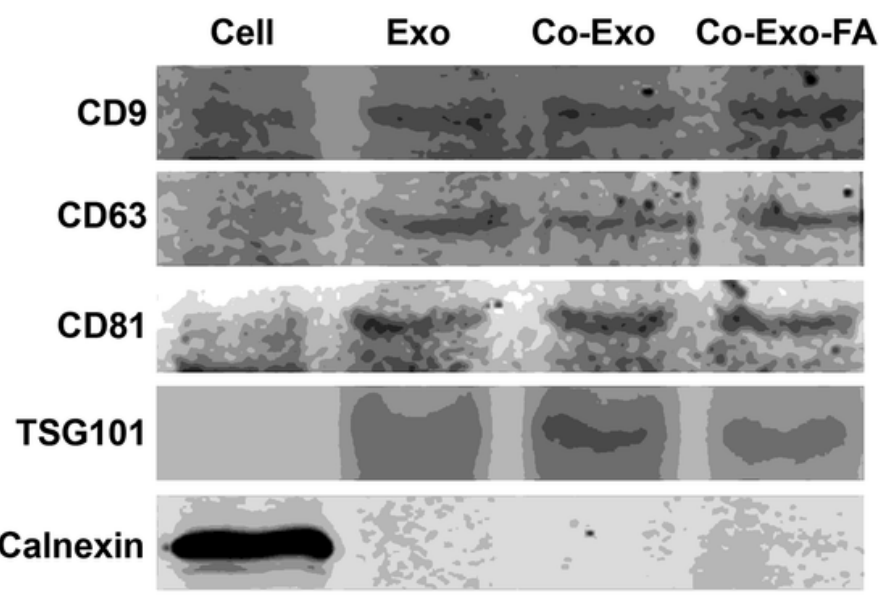

Figure 4

Characterization of Raw264.7 cell-derived exosomes. (A) Particle size and zeta potential of Exo, Co-Exo, and Co-Exo-FA determined by DLS ( $\mathrm{n}=3$ ). (B) Size distribution of Exo, Co-Exo, and Co-Exo-FA. (C) Representative TEM images of negatively stained Exo, Co-Exo and Co-Exo-FA. Scale bar is $100 \mathrm{~nm}$. (D) Western blotting analysis of cell lysates, Exo, Co-Exo, and Co-Exo-FA, showing expression of CD9, CD63, CD81, TSG101, and calnexin. Data are shown as mean \pm SD of three independent tests. ${ }^{*} P<0.01$. 

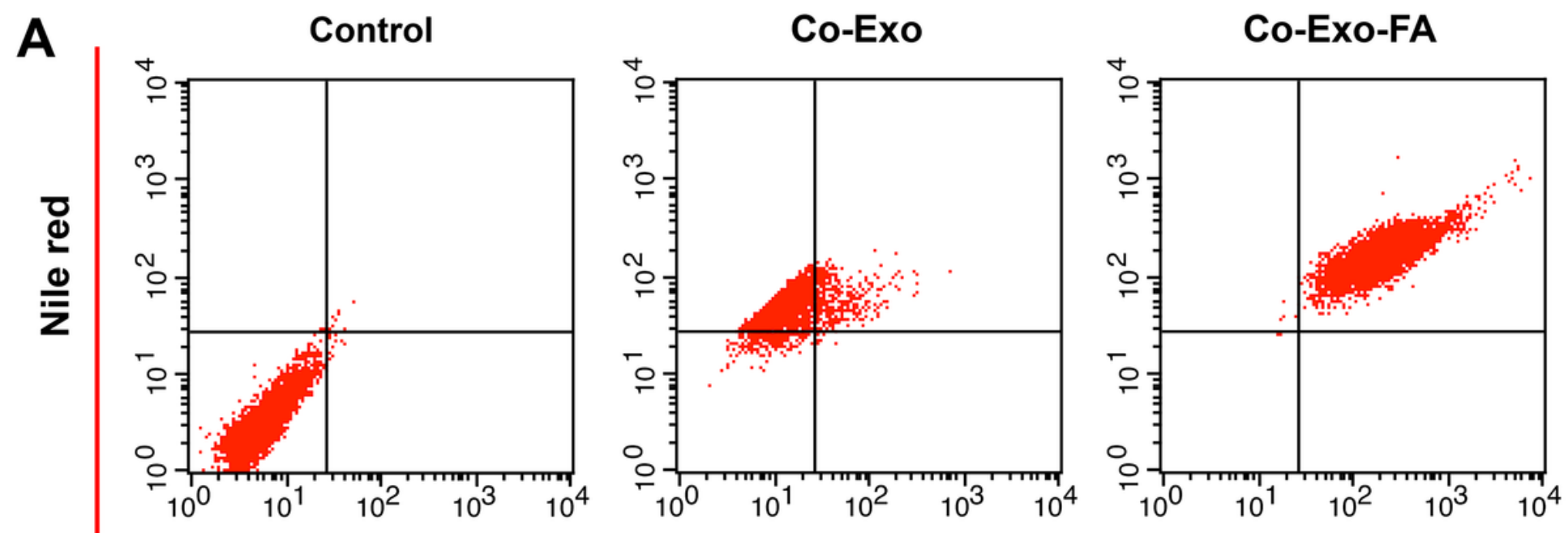

\section{FAM-siPLK1}

B
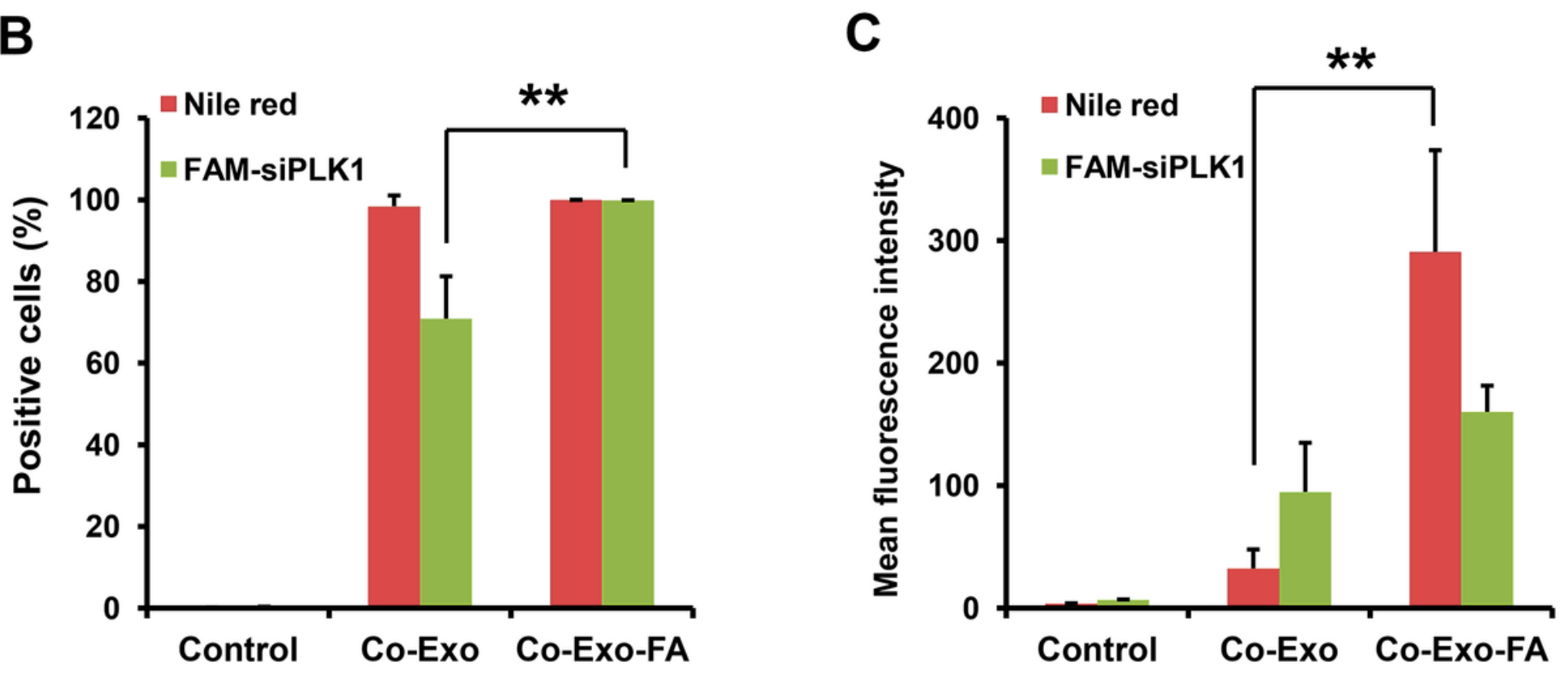

Figure 5

Flow cytometry data of Exo uptake by PC-3 cells. (A) Cellular uptake analysis of Co-Exo and Co-Exo-FA. (B) Quantitative analysis of the ratio of Nile and FAM-siRNA positive cells. (C) Mean fluorescence intensity of Nile and FAM-siRNA in PC-3 cells. ** $\mathrm{P}<0.01$. 


\section{DAPI}

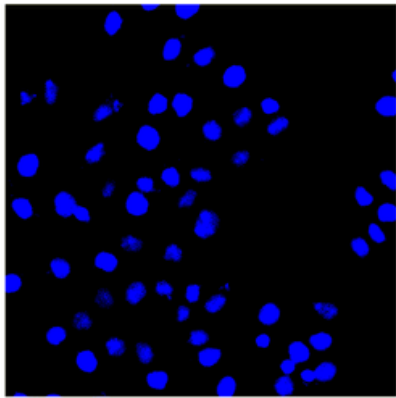

Nile red+

FAM-siPLK1

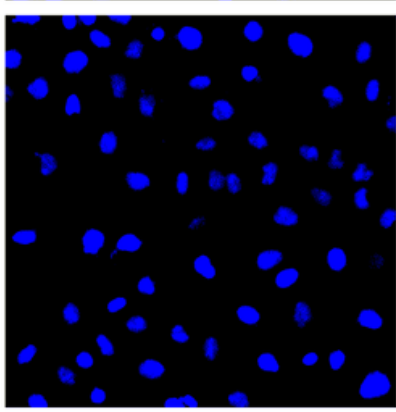

Co-Exo

Co-Exo-FA

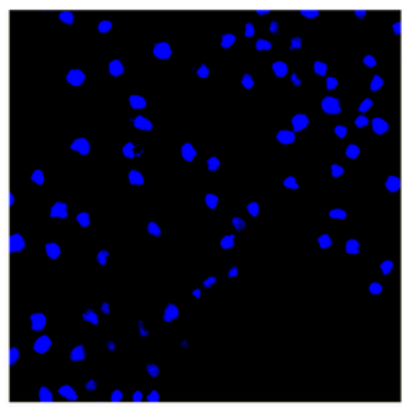

FAM
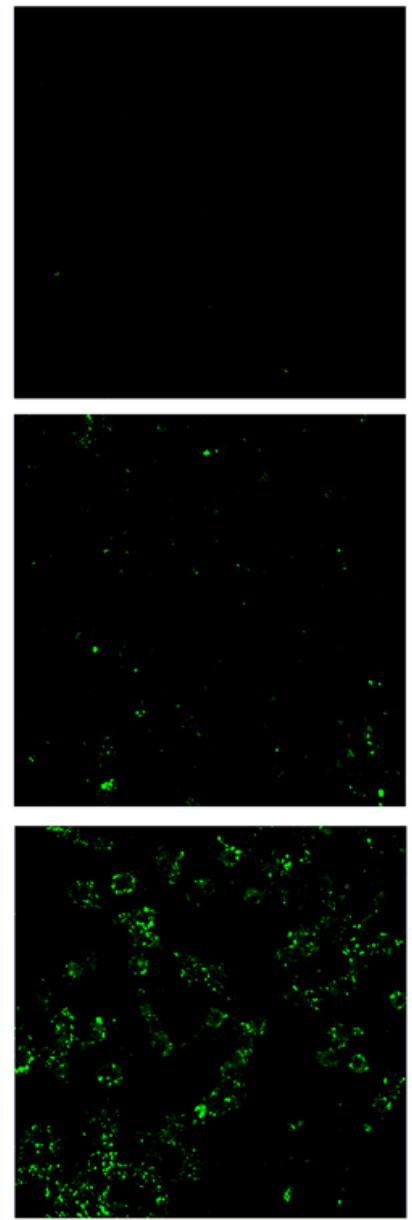

Nile red
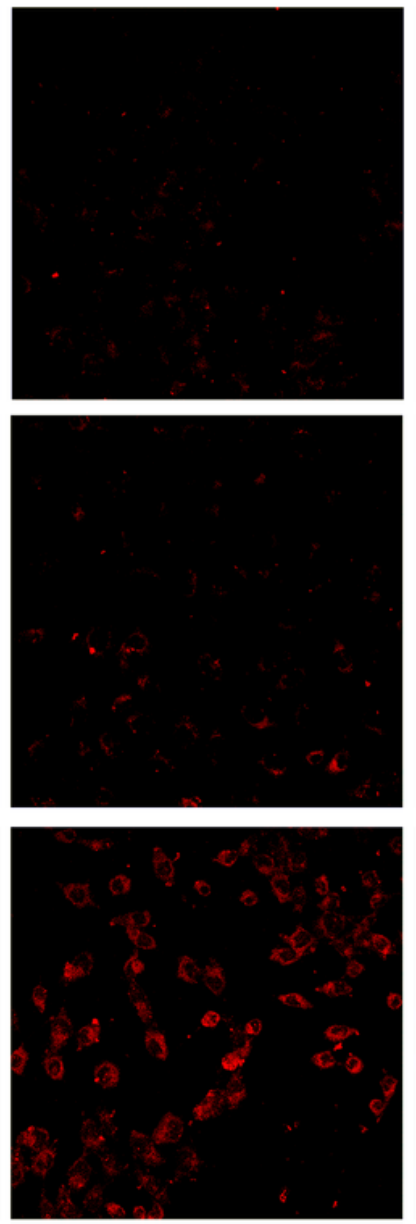

Merged
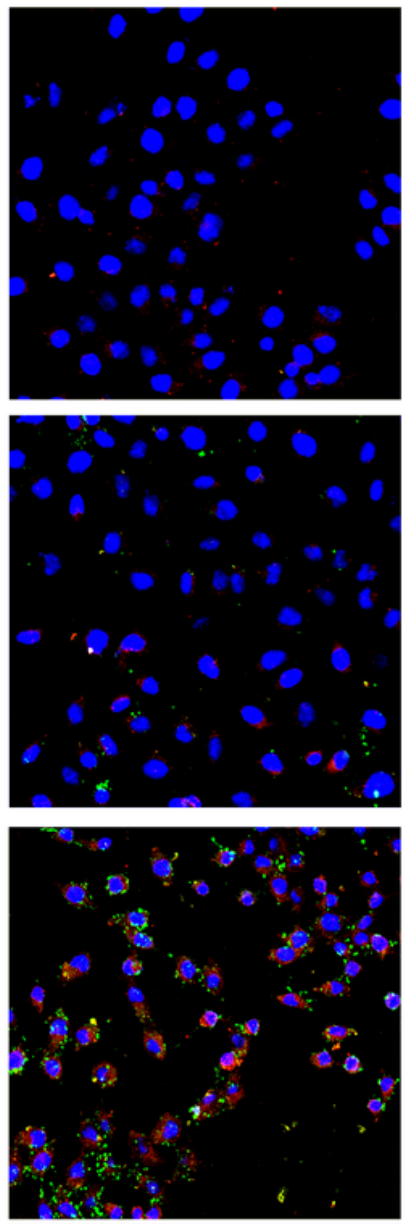

Figure 6

Confocal microscopy images of PC-3 cells after $3 \mathrm{~h}$ of incubation with Nile red and FAM-siRNA, Co-Exo, and Co-Exo-FA. Blue signals represent cell nucleus, red signals represent Nile red, and green signals represent FAM-siRNA. 

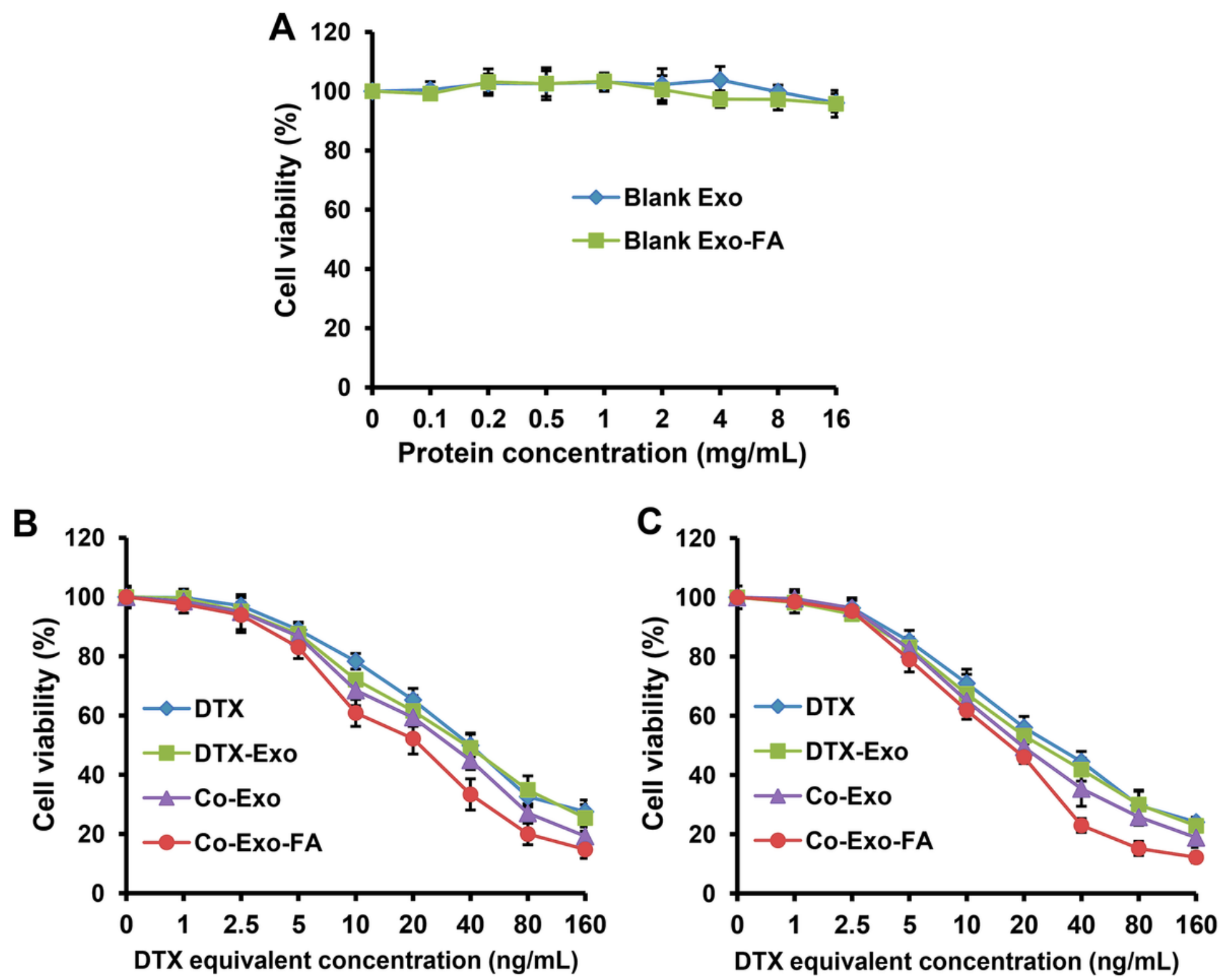

Figure 7

Effects of Co-Exo-FA on the proliferation of PC-3 and DU145 human prostate cancer cell lines in vitro. (A) Cytotoxicity of blank Exo and blank Exo-FA to PC-3 cells. (B) Dose dependent anti-proliferation assay of PC-3 cells for 48 h. (C) Dose dependent anti-proliferation assay of DU145 cells for $48 \mathrm{~h}$. 

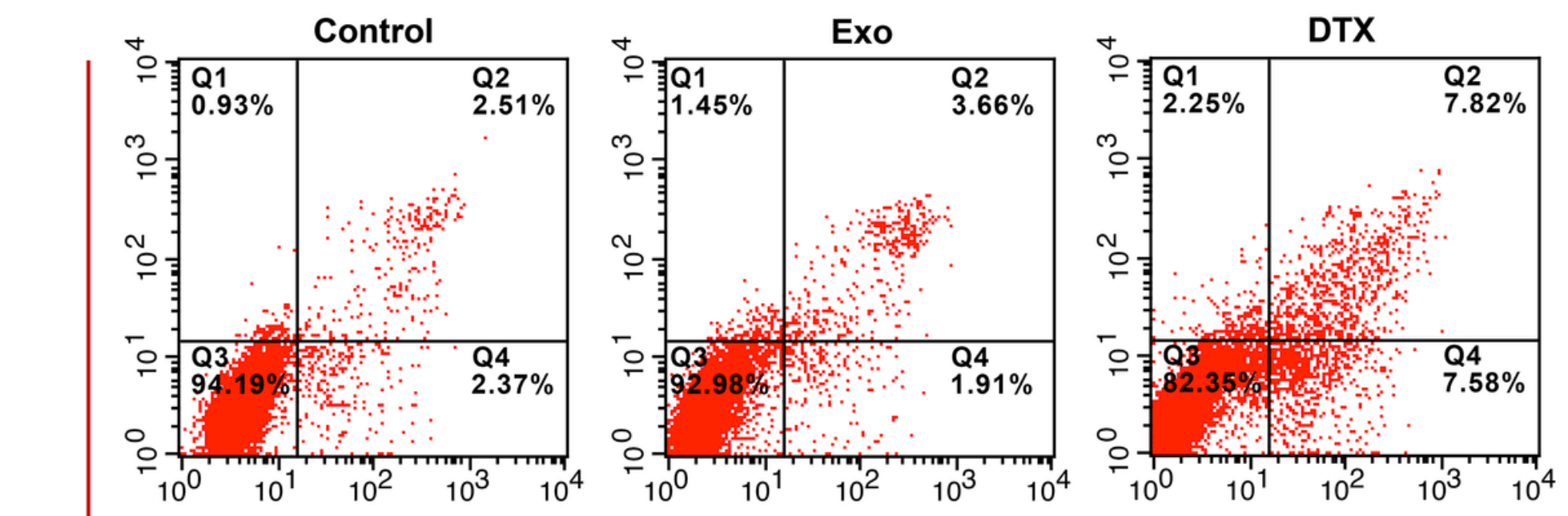

PI
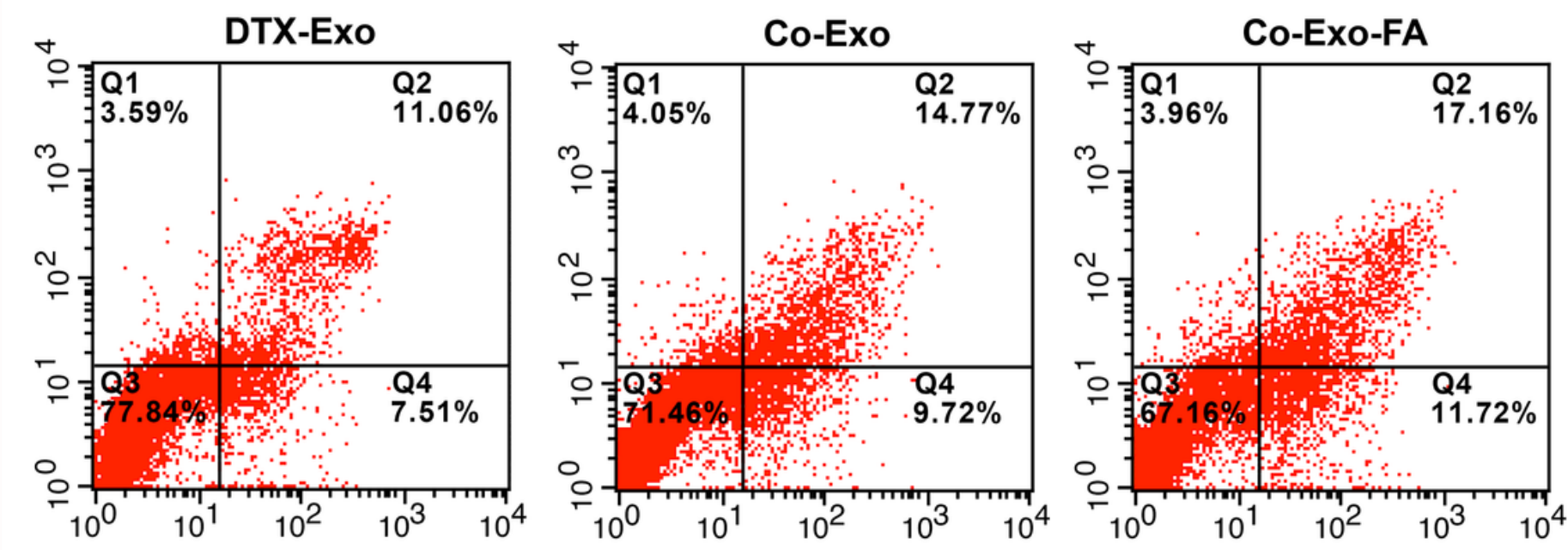

\section{Annexin V-FITC}

Figure 8

Cell apoptosis assay of PC-3 cells treated with different reagents for $24 \mathrm{~h}$ as measured by flow cytometry using Annexin V-FITC and PI staining. 
A

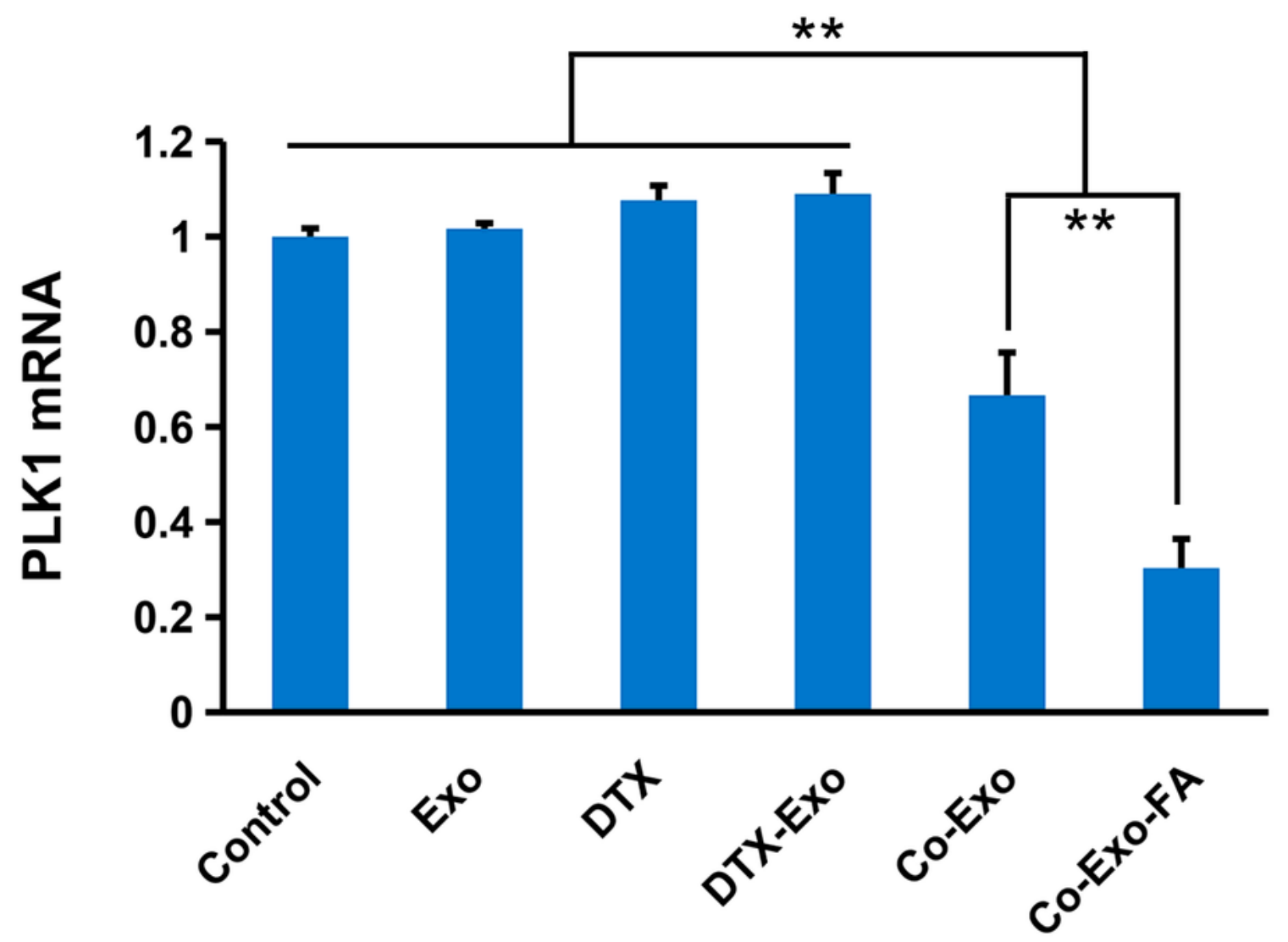

B

PLK1

GAPDH
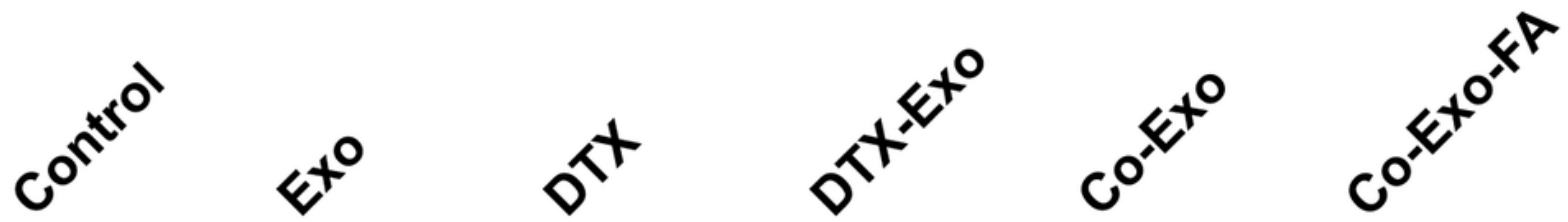

Figure 9

Relative mRNA levels of PLK1 (A) in PC-3 cells after treatment by different formulations. The expression of mRNA was measured by qRT-PCR. (B) The levels of PLK1 proteins were measured by western blotting analysis. Data are shown as mean \pm SD of three independent tests. ${ }^{\star \star} P<0.01$. 


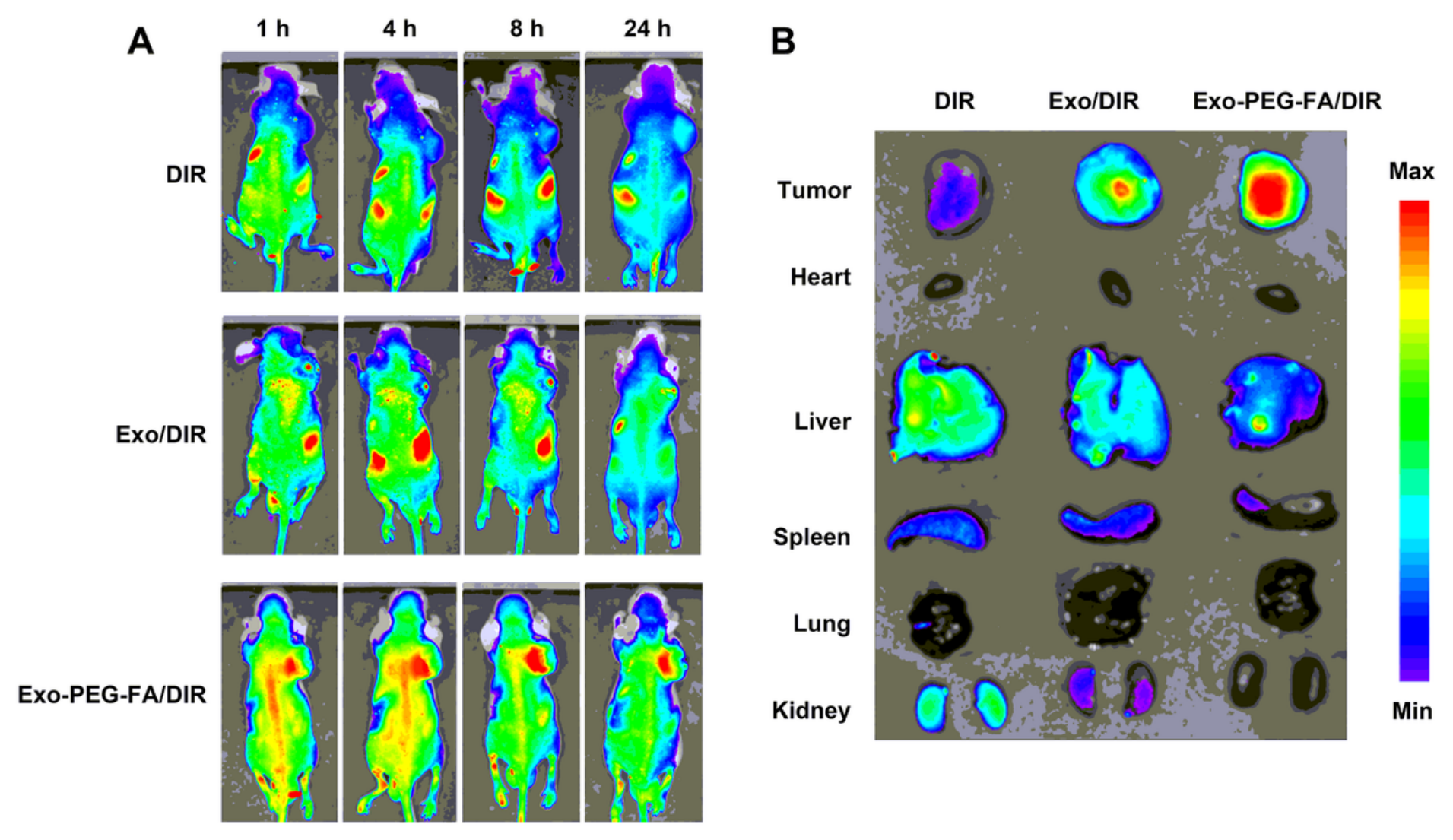

Figure 10

In vivo distribution of DIR, Co-Exo/DIR, and Co-Exo-FA/DIR. (A) Images were captured at 1, 4, 8, and $24 \mathrm{~h}$ after injection. (B) External imaging of the resected tumor and organs at the end of the experiment. Balb/c nude mice bearing xenograft tumors were injected with DIR, Co-Exo/DIR, or Co-Exo-FA/DIR via their tail vein. 

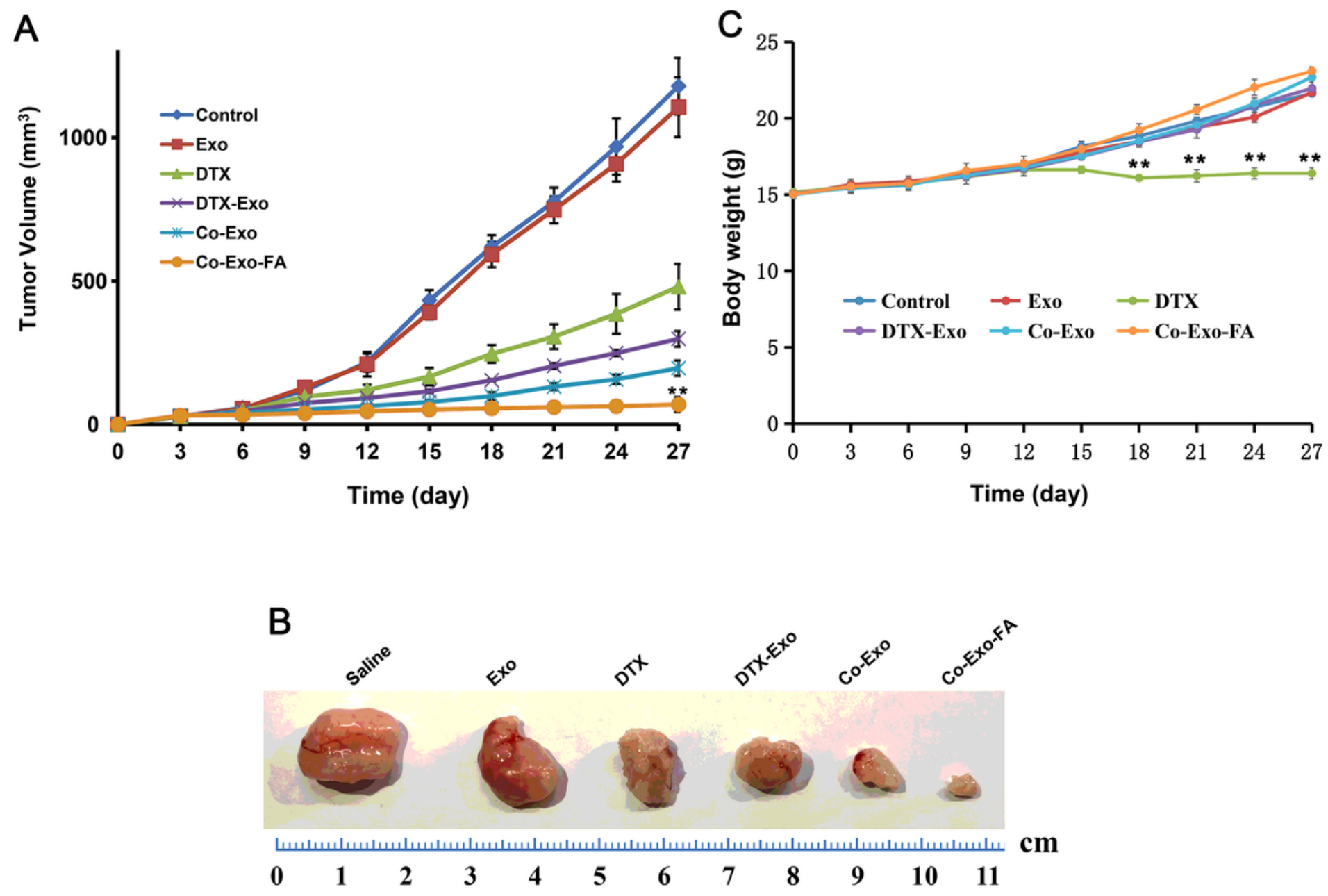

\section{Figure 11}

In vivo tumor growth suppression of Co-Exo-FA. (A) Observed responses in the volume of tumors after different treatments. (B) Representative images of isolated tumors at the end of the experiment. Balb/c nude mice bearing xenograft tumors were injected via their tail vein with either saline, blank Exo, DTX-Exo, Co-Exo, or Co-Exo-FA every $4 \mathrm{~d}$ for a total of 5 times. (C) Body weight changes of nude mice in each group during the experiment. Data are shown as mean \pm SD of six independent tests. $* * P<0.01$. 

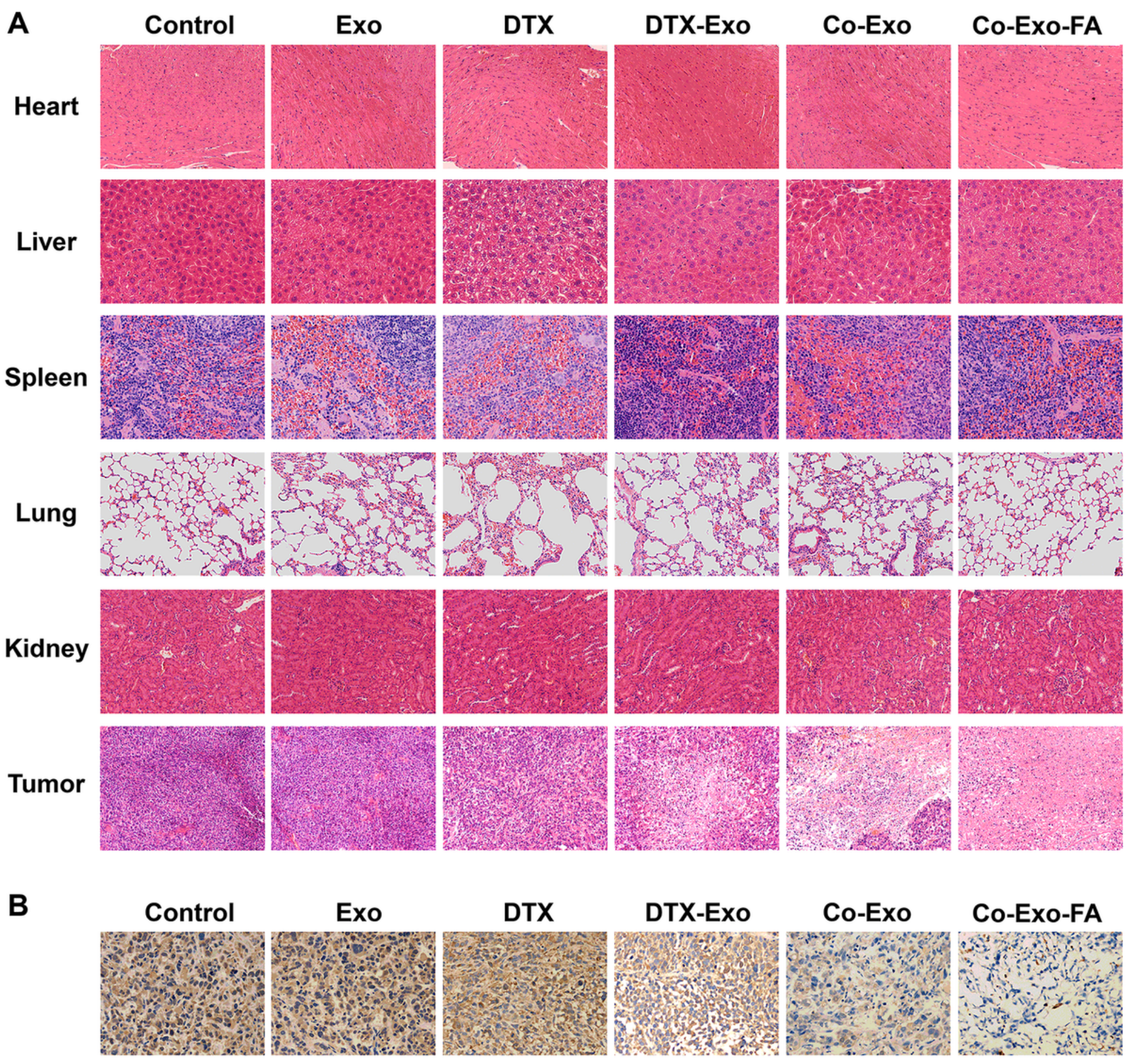

Figure 12

Histological analyses of tumors and organs by western blotting. (A) H\&E staining of tumors and organs. (B) IHC staining for detecting the expression of the PLK1 protein in tumor tissues. 